\title{
An Efficient Three-Party Authentication Scheme for Data Exchange in Medical Environment
}

\author{
Shin-Yan Chiou $\mathbb{D}^{1,2,3}$ and Ching-Hsuan Lin ${ }^{1}$ \\ ${ }^{1}$ Department of Electrical Engineering, College of Engineering, Chang Gung University, 259 Wen-Hwa 1st Road, \\ Kwei-Shan, Tao-Yuan, Taiwan \\ ${ }^{2}$ Department of Nuclear Medicine, Linkou Chang Gung Memorial Hospital, Tao-Yuan, Taiwan \\ ${ }^{3}$ Center for Biomedical Engineering, Chang Gung University, Tao-Yuan, Taiwan \\ Correspondence should be addressed to Shin-Yan Chiou; ansel@mail.cgu.edu.tw
}

Received 6 July 2017; Revised 17 October 2017; Accepted 10 December 2017; Published 2 January 2018

Academic Editor: Emanuele Maiorana

Copyright (c) 2018 Shin-Yan Chiou and Ching-Hsuan Lin. This is an open access article distributed under the Creative Commons Attribution License, which permits unrestricted use, distribution, and reproduction in any medium, provided the original work is properly cited.

\begin{abstract}
Compared with traditional paper medical records, electronic medical records have many advantages such as easy transmission, high efficiency, better accuracy, and easier storage. The further development and penetration of electronic medical records will raise increasingly critical transmitted-data accuracy and security issues. Previous studies have proposed a verifier-based three-party authentication scheme and to provide high efficiency and security, with low computation and transmission costs. However, this protocol fails to achieve anonymity, is vulnerable to tracking attacks, and is inefficient. In this paper, we propose a new authentication scheme which provides patient anonymity and resistance to tracking attacks, while reducing computation and communication costs. The proposed system is easier to implement and is more suitable for use in remote electronic medical record exchange systems.
\end{abstract}

\section{Introduction}

Aging societies experience increasing rates of chronic disease (e.g., heart disease, diabetes, cardiovascular diseases, and mental health issues) which must be frequently observed and monitored. Patients with such illnesses require periodic hospital- or clinic-based checkups, which can be inconvenient and stressful for elderly people. The digitization of medical measurement equipment (e.g., blood pressure monitors, blood glucose meters) allows for diagnostic information to be stored electronically and transmitted to remote locations for analysis and monitoring, enabling patients to avoid hospital visits while enabling their health care to closely monitor their status. Such electronic medical records (EMR) [1-3] has many advantages over conventional paper medical records, such as easy transmission, high efficiency, better accuracy, and ease of storage. However, their increased convenience raises significant security issues such as patient privacy and data integrity.

Telecare medicine information systems (TMIS) [4-6] involve the transmission of remote digital medical information or health reports through the combination of computers, communication systems to provide patients, and medical institutions with a secure data transmission platform and allow them to obtain medical record or health reports securely and conveniently. However, there are many security issues such as patient privacy and data integrity. Many identification and authentication protocols have been proposed to protect patient privacy and information. TMIS with threeparty authentication is a secure data transmission platform that allows an authentication server and two participants (a medical institution or doctor and a patient) to generate a session key and a secure channel to verify their identities and then exchange data securely.

Lin and Lee [7] proposed a verifier-based three-party authentication scheme to provide high efficiency and security, along with low computation and transmission costs. However, this protocol fails to achieve anonymity and is vulnerable to tracking attacks. In addition, when authenticating a participant, it takes considerable time for the server to locate the verifiers, making the system difficult to implement. We thus propose a new authentication scheme which provides 


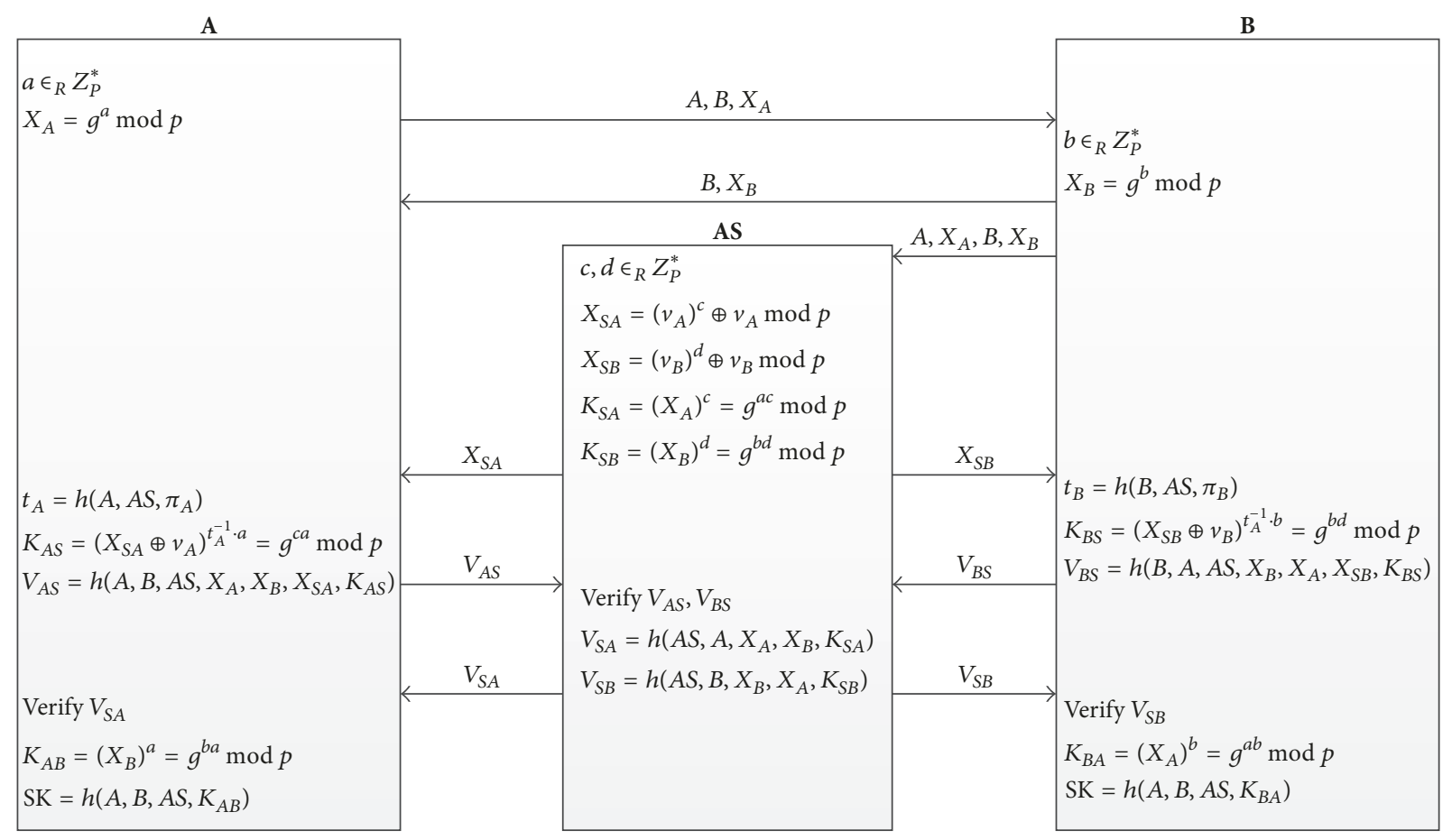

Figure 1: The procedure of Lee et al's scheme.

anonymity and resistance to tracking attacks, while reducing computation and communication costs. The proposed system is easier to implement and is more suitable for use in remote electronic medical record exchange systems.

The remainder of this paper is organized as follows. Section 2 reviews and analyzes verifier-based three-party authentication schemes without server public key including Lee et al.s [8], Wang-Mo's [9], Kwon et al.s [10], and LinLee's [7] schemes. Section 3 introduces notations and security requirements for our scheme. Section 4 presents our proposed protocol, and the security analysis is given in Section 5. Section 6 provides a comparison of the proposed protocol and other related works. Finally, an implementation is described in Section 7 and a conclusion is drawn in Section 8 .

\section{Related Works}

This section reviews four verifier-based three-party authentication schemes without server public key including Lee et al.s [8], Wang-Mo's [9], Kwon et al.s [10], and Lin-Lee's [7] schemes and analyzes the weaknesses of their schemes.

2.1. Review of Lee et al.'s Scheme. Lee et al. [8] proposed a verifier-based authentication scheme without server's public key based on the Diffie-Hellman key exchange. Their scheme enables each client to only remember a memorable password. The normal procedure of their scheme is shown in Figure 1.

2.1.1. Initialization Phase. A client $A$ and a trusted authentication server $A S$ share a verifier $v_{A}=g^{t_{A}} \bmod p$ for a password $\pi_{A}$, and a client $B$ and $A S$ share a verifiers $v_{B}=$ $g^{t_{B}} \bmod p$ for a password $\pi_{B}$, where $t_{A}=h\left(A, A S, \pi_{A}\right)$ and $t_{B}=h\left(A, A S, \pi_{B}\right)$.

2.1.2. Verification Phase. This phase allows $A$ and $B$ to share a secret key confidentially via $A S$. The details of the execution steps are described as follows (Figure 1).

(1) $A \rightarrow B: A, B, X_{A}: A$ chooses a random number $a \epsilon_{R} Z_{P}^{*}$, computes $X_{A}=g^{a} \bmod p$, and sends $A, B$, $X_{A}$ to $B$.

(2) $B \rightarrow A: B, X_{B}$ and $B \rightarrow A S: A, X_{A}, B, X_{B}: B$ chooses a random number $b \epsilon_{R} Z_{P}^{*}$, computes $X_{B}=g^{b} \bmod$ $p$, and sends $\left(B, X_{B}\right)$ and $\left(A, X_{A}, B, X_{B}\right)$ to $A$ and $A S$, respectively.

(3) $A S \rightarrow A: X_{S A}$ and $A S \rightarrow B: X_{S B}$ : after receiving $v_{a}$ and $v_{b}, A S$ chooses two random numbers $c, d \in_{R} Z_{P}^{*}$, computes $X_{S A}=\left(v_{A}\right)^{c} \oplus v_{A} \bmod p, X_{S B}=\left(v_{B}\right)^{d} \oplus$ $v_{B} \bmod p, K_{S A}=\left(X_{A}\right)^{c}=g^{a c} \bmod p$, and $K_{S B}=$ $\left(X_{B}\right)^{d}=g^{b d} \bmod p$, and sends $X_{S A}$ and $X_{S B}$ to $A$ and $B$, respectively.

(4) $A \rightarrow A S: V_{A S}$ and $B \rightarrow A S: V_{B S}: A$ computes $K_{A S}=\left(X_{S A} \oplus v_{A}\right)^{t_{A}^{-1} \cdot a}=g^{c a} \bmod p$ and $V_{A S}=h\left(A, B, A S, X_{A}, X_{B}, X_{S A}, K_{A S}\right)$, sends $V_{A S}$ to $A S$, where $t_{A}=h\left(A, A S, \pi_{A}\right)$. B computes $K_{B S}=\left(X_{S B} \oplus v_{B}\right)^{t_{B}^{-1} \cdot b}=g^{b d} \bmod p$ and $V_{B S}=$ $h\left(B, A, A S, X_{B}, X_{A}, X_{S B}, K_{B S}\right)$, and sends $V_{B S}$ to $A S$, where $t_{B}=h\left(B, A S, \pi_{B}\right)$.

(5) $A S \rightarrow A: V_{S A}$ and $A S \rightarrow B: V_{S B}: A S$ verifies whether $V_{A S}$ and $V_{B S}$ are valid. If they do, $A S$ 


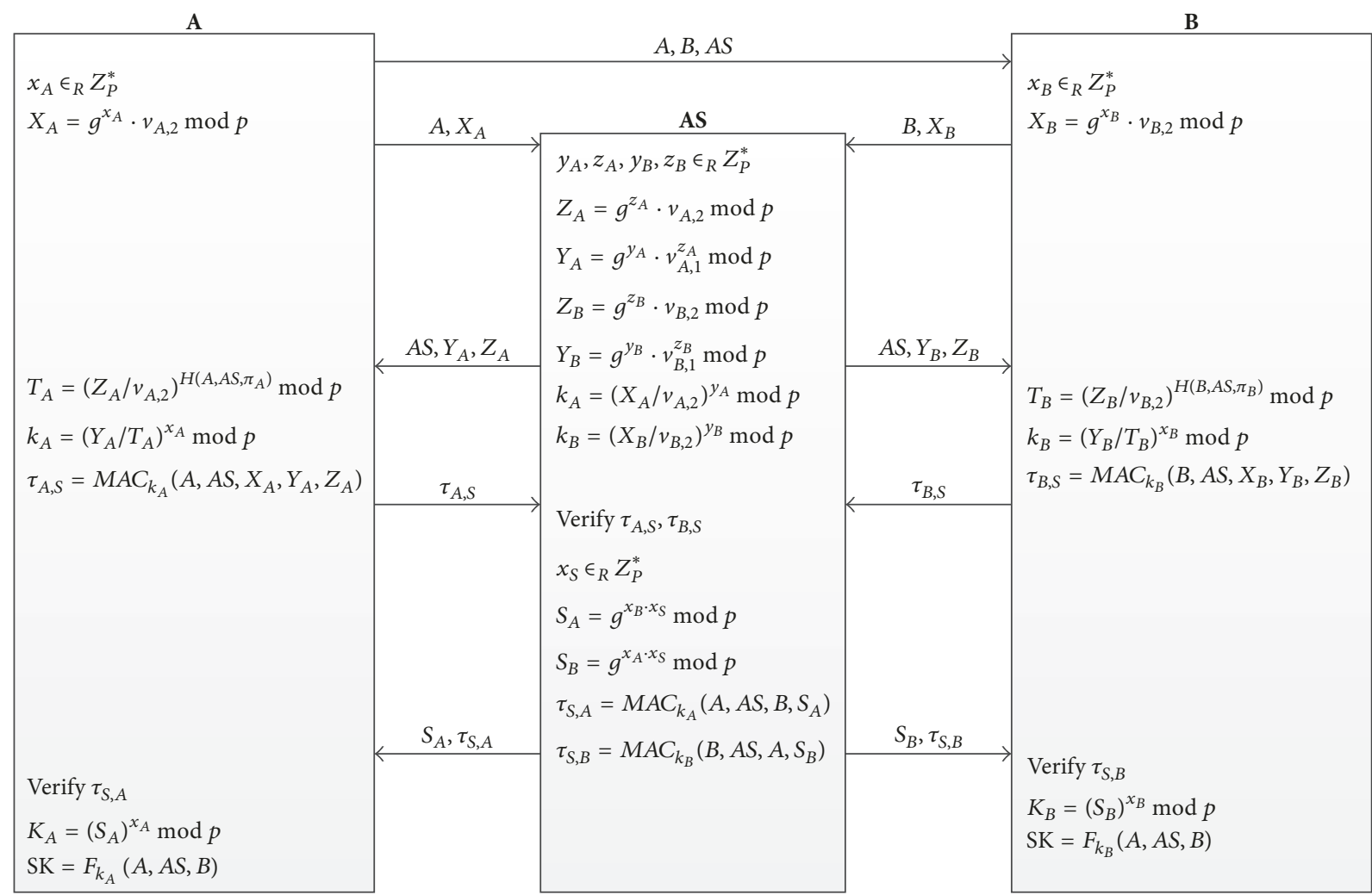

FIgURE 2: The procedure of Kwon et al's scheme.

computes $V_{S A}=h\left(A S, A, X_{A}, X_{B}, K_{S A}\right)$ and $V_{S B}=$ $h\left(A S, B, X_{B}, X_{A}, K_{S B}\right)$ and sends $V_{S A}$ and $V_{S B}$ to $A$ and $B$, respectively.

(6) $A$ verifies $V_{S A}$ and $B$ verifies $V_{S B}$.

Finally, $A$ possesses a session key $K_{A B}=\left(X_{B}\right)^{a}=$ $g^{b a} \bmod p$ and $B$ possesses a session key $K_{B A}=\left(X_{A}\right)^{b}=$ $g^{a b} \bmod p$.

2.1.3. Weaknesses of Lee et al.'s Scheme. Wang and Mo [9] showed that the Lee et al.s scheme [8] is not resistant to an impersonation attack if an attacker once has stolen $A$ 's verifier $v_{A}$.

2.2. Review of Wang-Mo's Scheme. In order to withstand an impersonation attack of the Lee et al.s scheme [8] under verifier-stolen situation, Wang and Mo [9] modified $V_{A S}$ as $h\left(A, B, A S, X_{A}, X_{B},\left(X_{S A} \oplus v_{A}\right)^{t_{A}^{-1}}=g^{c}, K_{A S}\right)$ and $V_{B S}$ as $h\left(B, A, A S, X_{B}, X_{A},\left(X_{S B} \oplus v_{B}\right)^{t_{B}^{-1}}=g^{d}, K_{B S}\right)$, respectively. Therefore, if both verifiers $V_{A S}$ and $V_{B S}$ are stole, an impersonation attack does not work without $t_{A}$ and $t_{B}$.

However, Lin and Lee [7] showed that the Wang and Mo's scheme [9] do not realize key confirmation. If the transmitted EMRs or EHRs are encrypted by using an unconfirmed key, their integrity and confidentiality are unsure.

2.3. Review of Kwon et al.'s Scheme. This section reviews Kwon et al.'s scheme [10]. The normal procedure of their scheme is shown in Figure 2.
2.3.1. Initialization Phase. $A$ and $A S$ share a verifier $v_{A, 1}=$ $g^{t_{A}} \bmod p$ and $v_{A, 2}=g_{2}^{t_{A}} \bmod p$ for a password $\pi_{A}$, and $B$ and $A S$ share a verifiers $v_{B, 1}=g^{t_{B}} \bmod p$ and $v_{B, 2}=$ $g_{2}^{t_{B}} \bmod p$ for a password $\pi_{B}$, where $t_{A}=h\left(A, A S, \pi_{A}\right)$ and $t_{B}=h\left(A, A S, \pi_{B}\right)$.

2.3.2. Verification Phase. This phase allows $A$ and $B$ to share a secret key confidentially via $A S$. The details of the execution steps are described as follows (Figure 2):

(1) $A \rightarrow B: A, B, A S: A$ broadcasts $(A, B, A S)$.

(2) $A \rightarrow A S: A, X_{A}$ and $B \rightarrow A S: B, X_{B}: A$ chooses a random number $x_{A} \in_{R} Z_{P}^{*}$, computes $X_{A}=g^{x_{A}}$. $v_{A, 2} \bmod p$, and sends $\left(A, X_{A}\right)$ to $A S$. $B$ chooses a random number $x_{B} \in_{R} Z_{P}^{*}$, computes $X_{B}=g^{x_{B}}$. $v_{B, 2} \bmod p$, and sends $\left(B, X_{B}\right)$ to $A S$.

(3) $A S \rightarrow A: A S, Y_{A}, Z_{A}$ and $A S \rightarrow B: A S, Y_{B}, Z_{B}$ : $A S$ chooses random numbers $y_{A}, z_{A}, y_{B}, z_{B} \in_{R} Z_{P}^{*}$, computes $Z_{A}=g^{z_{A}} \cdot v_{A, 2} \bmod p, Y_{A}=g^{y_{A}}$. $v_{A, 1}^{z_{A}} \bmod p, Z_{B}=g^{z_{B}} \cdot v_{B, 2} \bmod p$ and $Y_{B}=g^{y_{B}}$. $v_{B, 1}^{z_{B}} \bmod p$, and sends $\left(A S, Y_{A}, Z_{A}\right)$ and $\left(A S, Y_{B}, Z_{B}\right)$ to $A$ and $B$, respectively. Moreover, $A S$ computes $k_{A}=$ $\left(X_{A} / v_{A, 2}\right)^{y_{A}} \bmod p$ and $k_{B}=\left(X_{B} / v_{B, 2}\right)^{y_{B}} \bmod p$.

(4) $A \rightarrow A S: \tau_{A, S}$ and $B \rightarrow A S: \tau_{B, S}$ : after receiving $\left(A S, Y_{A}, Z_{A}\right), A$ computes $T_{A}=\left(Z_{A} /\right.$ $\left.v_{A, 2}\right)^{H\left(A, A S, \pi_{A}\right)} \bmod p, k_{A}=\left(Y_{A} / T_{A}\right)^{x_{A}} \bmod p$, and $\tau_{A, S}=M A C_{k_{A}}\left(A, A S, X_{A}, Y_{A}, Z_{A}\right)$ and sends $\tau_{A, S}$ to 


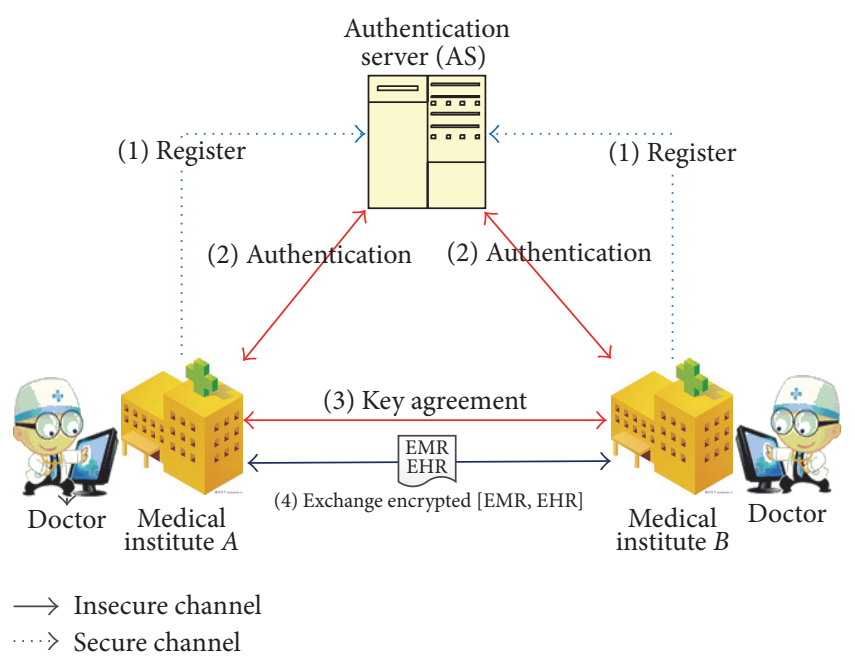

FIgURE 3: The normal procedure of Lin-Lee's scheme.

AS. After receiving $\left(A S, Y_{B}, Z_{B}\right), B$ computes $T_{B}=$ $\left(Z_{B} / v_{B, 2}\right)^{H\left(B, A S, \pi_{B}\right)} \bmod p, k_{B}=\left(Y_{B} / T_{B}\right)^{x_{B}} \bmod p$, and $\tau_{B, S}=M A C_{k_{B}}\left(B, A S, X_{B}, Y_{B}, Z_{B}\right)$ and sends $\tau_{B, S}$ to $A S$.

(5) $A S \rightarrow A: S_{A}, \tau_{S, A}$ and $A S \rightarrow B: S_{B}, \tau_{S, B}: A S$ verifies whether $\tau_{A, S}$ and $\tau_{B, S}$ are correct. If so, $A S$ chooses a random number $x_{S} \in_{R} Z_{P}^{*}$, computes $S_{A}=g^{x_{B} \cdot x_{S}} \bmod$ $p, S_{B}=g^{x_{A} \cdot x_{S}} \bmod p, \tau_{S, A}=M A C_{k_{A}}\left(A, A S, B, S_{A}\right)$, and $\tau_{S, B}=\operatorname{MAC}_{k_{B}}\left(B, A S, A, S_{B}\right)$, and sends $\left(S_{A}, \tau_{S, A}\right)$ and $\left(S_{B}, \tau_{S, B}\right)$ to $A$ and $B$, respectively.

(6) $A$ verifies $\tau_{S, A}$ and $B$ verifies $\tau_{S, B}$.

Finally, $A$ possesses a session key $K_{A}=\left(S_{A}\right)^{x_{A}} \bmod p$ and $B$ possesses a session key $K_{B}=\left(S_{B}\right)^{x_{B}} \bmod p$.

2.3.3. Weaknesses of Kwon et al.'s Scheme. Lin and Lee [7] showed that the Kwon et al.s scheme [10] do not realize key confirmation. If the transmitted EMRs or EHRs are encrypted by using an unconfirmed key, their integrity and confidentiality are unsure.

2.4. Review of Lin-Lee's Scheme. This section reviews LinLee's scheme [7] (including the initialization and verification phases). The normal procedure of their scheme is shown in Figures 3 and 4 .

$A$ and $B$ represent two medical institutions, and $A S$ stands for a trusted authentication server.

2.4.1. Initialization Phase. As shown in steps (1) in Figure 3, $A$ and $B$ send their verifiers to $A S$ via a secure and verified channel to register their verifiers. The details of the steps are as follows: $A$ sends verifier $v_{A}=g^{t_{A}} \bmod p$ to $A S$, and $B$ sends verifier $v_{B}=g^{t_{B}} \bmod p$ to $A S$, where $t_{A}=H\left(\mathrm{ID}_{A}, \mathrm{ID}_{A S}, \pi_{A}\right)$ and $t_{B}=H\left(\mathrm{ID}_{B}, \mathrm{ID}_{A S}, \pi_{B}\right)$.

2.4.2. Verification Phase. Assume $A$ and $B$ need to exchange EMR or EHR confidentially via authentication server $A S$. As shown in steps (2), (3), and (4) in Figure 3, $A$ and $B$ process a mutual authentication with $A S$ and each other, perform a key agreement to obtain a session key, and exchange encrypted EMR or EHR encrypted by the session key. The details of the execution steps are described as follows:

(1) $A \rightarrow A S: \mathrm{ID}_{A}, \mathrm{ID}_{B}, X_{A}$ :A chooses a random number $a \in_{R} Z_{P}^{*}$, computes $X_{A}=g^{a} \bmod p$, and sends $\mathrm{ID}_{A}$, $\mathrm{ID}_{B}, X_{A}$ to $A S$.

(2) $A \rightarrow B: \mathrm{ID}_{A}, X_{A}: A$ sends $\mathrm{ID}_{A}$ and $X_{A}$ to $B$.

(3) $A S \rightarrow A: X_{S A}$, AS $\rightarrow B: X_{S B}: A S$ chooses two random numbers $c, d \in_{R} Z_{P}^{*}$, computes $X_{S A}=\left(v_{A}\right)^{c} \oplus$ $v_{A} \bmod p$ and $X_{S B}=\left(v_{B}\right)^{d} \oplus v_{B} \bmod p$, sends $X_{S A}$ to $A$, and sends $X_{S B}$ to $B$.

(4) $B \rightarrow A S: \mathrm{ID}_{A}, \mathrm{ID}_{B}, X_{B}: B$ chooses a random number $b \in_{R} Z_{P}^{*}$, computes $X_{B}=g^{b} \bmod p$, and sends $\mathrm{ID}_{A}$, $\mathrm{ID}_{B}$, and $X_{B}$ to $A S$.

(5) AS: AS computes $g^{c}, g^{d}, K_{S A}=\left(X_{A}\right)^{c}=g^{a c} \bmod p$, and $K_{S B}=\left(X_{B}\right)^{d}=g^{b d} \bmod p$.

(6) $B \rightarrow A: \mathrm{ID}_{B}, X_{B}: B$ sends $\mathrm{ID}_{B}, X_{B}$ to $A$.

(7) $A \rightarrow A S: V_{A S}: A$ computes $g^{c}=\left(X_{S A} \oplus v_{A}\right)^{t_{A}^{-1}} \bmod p$, uses $g^{c}$ and $a$ to evaluate $K_{A S}=\left(g^{c}\right)^{a}=g^{c a} \bmod$ $p, V_{A S}=h\left(\mathrm{ID}_{A}, \mathrm{ID}_{B}, \mathrm{ID}_{A S}, X_{A}, X_{B}, g^{c}, K_{A S}\right), K_{A B}=$ $\left(X_{B}\right)^{a}=g^{b a} \bmod p$, and $\mu_{A B}=h\left(\mathrm{ID}_{A}, \mathrm{ID}_{B}, X_{A}\right.$, $\left.X_{B}, K_{A B}\right)$, and sends $V_{A S}$ to $A S$.

(8) $B \rightarrow A S: V_{B S}: B$ computes $g^{d}=\left(X_{S B} \oplus v_{B}\right)^{t_{B}^{-1}} \bmod p$, uses $g^{d}$ and $b$ to evaluate $K_{B S}=\left(g^{d}\right)^{b}=g^{d b} \bmod$ $p, V_{B S}=h\left(\mathrm{ID}_{A}, \mathrm{ID}_{B}, \mathrm{ID}_{A S}, X_{A}, X_{B}, g^{d}, K_{B S}\right), K_{B A}=$ $\left(X_{A}\right)^{b}=g^{a b} \bmod p$, and $\mu_{A B}=h\left(\mathrm{ID}_{A}, \mathrm{ID}_{B}, X_{A}\right.$, $\left.X_{B}, K_{A B}\right)$, and sends $V_{B S}$ to $A S$.

(9) $A \rightarrow B: \mu_{A B}: A$ sends $\mu_{A B}=h\left(\mathrm{ID}_{A}, \mathrm{ID}_{B}, X_{A}\right.$, $\left.X_{B}, K_{A B}\right)$ to $B$.

(10) $B$ authenticates $A$ : $B$ verifiers whether the values $\mathrm{ID}_{A}, \mathrm{ID}_{B}, X_{A}, X_{B}, K_{A B}$ in $\mu_{A B}$ are correct. If so, the identity of $A$ is valid.

(11) $B \rightarrow A: \mu_{B A}: B$ sends $\mu_{B A}=h\left(\mathrm{ID}_{A}, \mathrm{ID}_{B}, X_{A}\right.$, $\left.X_{B}, K_{B A}\right)$ to $A$.

(12) $A$ authenticates $B: A$ verifiers whether the values $\mathrm{ID}_{A}, \mathrm{ID}_{B}, X_{A}, X_{B}, K_{B A}$ in $\mu_{B A}$ are correct. If so, the identity of $B$ is valid.

(13) $A S$ authenticates $A, B: A S$ verifiers whether the equations $V_{A S}=h\left(\mathrm{ID}_{A}, \mathrm{ID}_{B}, \mathrm{ID}_{A S}, X_{A}, X_{B}, g^{c}, K_{A S}\right)$ and $V_{B S}=h\left(\mathrm{ID}_{A}, \mathrm{ID}_{B}, \mathrm{ID}_{A S}, X_{A}, X_{B}, g^{d}, K_{B S}\right)$ are hold. If so, the identities of $A$ and $B$ are valid.

(14) $A S \rightarrow A: V_{S A}$; $A S \rightarrow B: V_{S B}: A S$ computes $V_{S A}=h\left(\mathrm{ID}_{A S}, \mathrm{ID}_{A}, X_{A}, X_{B}, K_{S A}\right)$ and $V_{S B}=$ $h\left(\mathrm{ID}_{A S}, \mathrm{ID}_{B}, X_{B}, X_{A}, K_{S B}\right)$, sends $V_{S A}$ to $A$, and sends $V_{S B}$ to $B$.

(15) $A$ authenticates $A S$ : $A$ verifiers whether $V_{S A}$ is correct. If so, the identity of $A S$ is valid.

(16) $B$ authenticates $A S$ : $B$ verifiers whether $V_{S B}$ is correct. If so, the identity of $A S$ is valid.

Finally, $A$ possesses a session key $\mathrm{SK}_{A B}=h\left(\mathrm{ID}_{A}, \mathrm{ID}_{B}\right.$, $\left.\mathrm{ID}_{A S}, K_{A B}\right)$ and $B$ possesses a session key $\mathrm{SK}_{B A}=h\left(\mathrm{ID}_{A}\right.$, $\left.\mathrm{ID}_{B}, \mathrm{ID}_{A S}, K_{B A}\right)$. 


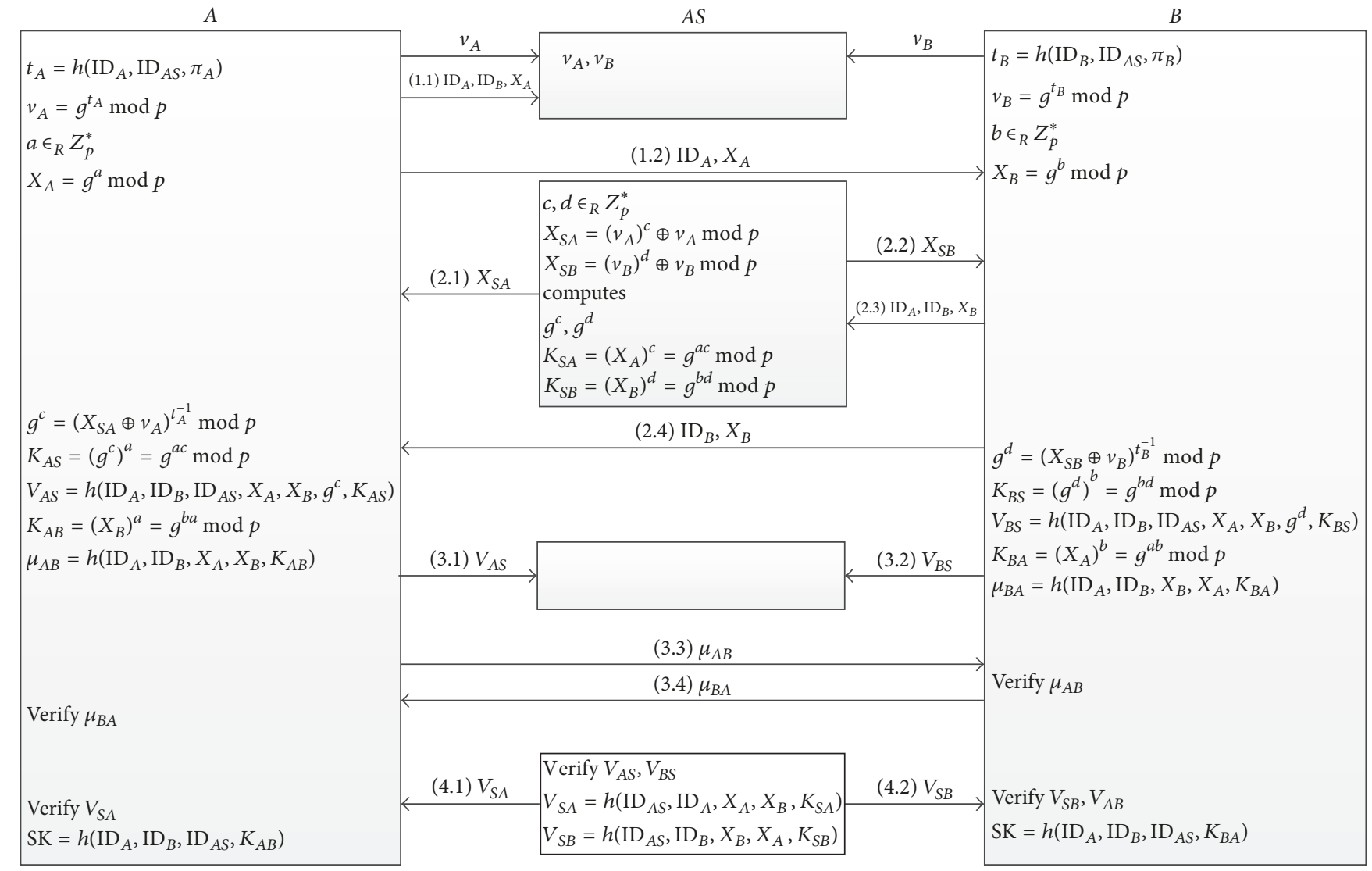

FIGURE 4: The procedure of Lin-Lee's scheme.

2.4.3. Weaknesses of Lin-Lee's Scheme. We find Lin-Lee's scheme [7] has three drawbacks: (1) does not provide anonymity, (2) vulnerable to tracking attack, and (3) inefficiency.

(1) Does Not Provide Anonymity. Medical institution A transmits data $\mathrm{ID}_{A}$ and $\mathrm{ID}_{B}$ to $A S$ in the step (1) of verification phase, while medical institution $B$ transmits the same data $\left(\mathrm{ID}_{A}\right.$ and $\left.\mathrm{ID}_{B}\right)$ to $A S$ in the step (4) of verification phase. An attacker can obtain the identity $\left(\mathrm{ID}_{A}\right.$ and $\left.\mathrm{ID}_{B}\right)$ of both $A$ and $B$ by eavesdropping on the transmission; thus the scheme does not provide anonymity.

(2) Vulnerable to Tracking Attack. The data $\mathrm{ID}_{A}$ and $\mathrm{ID}_{B}$ are transmitted in both step (1) and step (4) of verification phase. An adversary can track institutions $A$ and $B$ easily from the identity $\left(\mathrm{ID}_{A}\right.$ and $\left.\mathrm{ID}_{B}\right)$; thus the scheme is vulnerable to tracking attack.

(3) Inefficiency. The scheme needs 16 exponentiations; therefore the computation cost of Lin-Lee's scheme is inefficiency.

\section{Preliminary}

3.1. Notations. Notations section shows the notations used in our protocol, where $\operatorname{SID}_{X}=h\left(\operatorname{ID}_{X}, \pi_{X}, T_{X}\right)$ is the pseudo ID of $X, D_{U V}$ is a data encrypted by $V$ using symmetric key encryption algorithm and sent to $U$ from $V$, and $X, U$, and $V$ represent medical institutions $A, B$, or server $S$.

3.2. Attacker Model. In our scheme, we assume that the channels between $A$ and $S, B$ and $S$, and $A$ and $B$ are insecure. Any identity communicates with each other via an insecure public channel, offering adversaries opportunities to intercept. In the following, we present the assumptions of the attacker model [18-21].

(1) An adversary may eavesdrop on all communications between protocol actors over the public channel.

(2) An attacker can modify, delete, resend, and reroute the eavesdropped message.

(3) An attacker cannot be a legitimate server.

(4) The attacker knows the protocol description, which means the protocol is public.

3.3. Security Requirements. The security requirements of our proposed scheme are listed as follows:

(1) Data integrity: an adversary cannot alter the transmitted data without being detected.

(2) Anonymity: an adversary cannot know the identities of medical institutions through the eavesdropped data. 


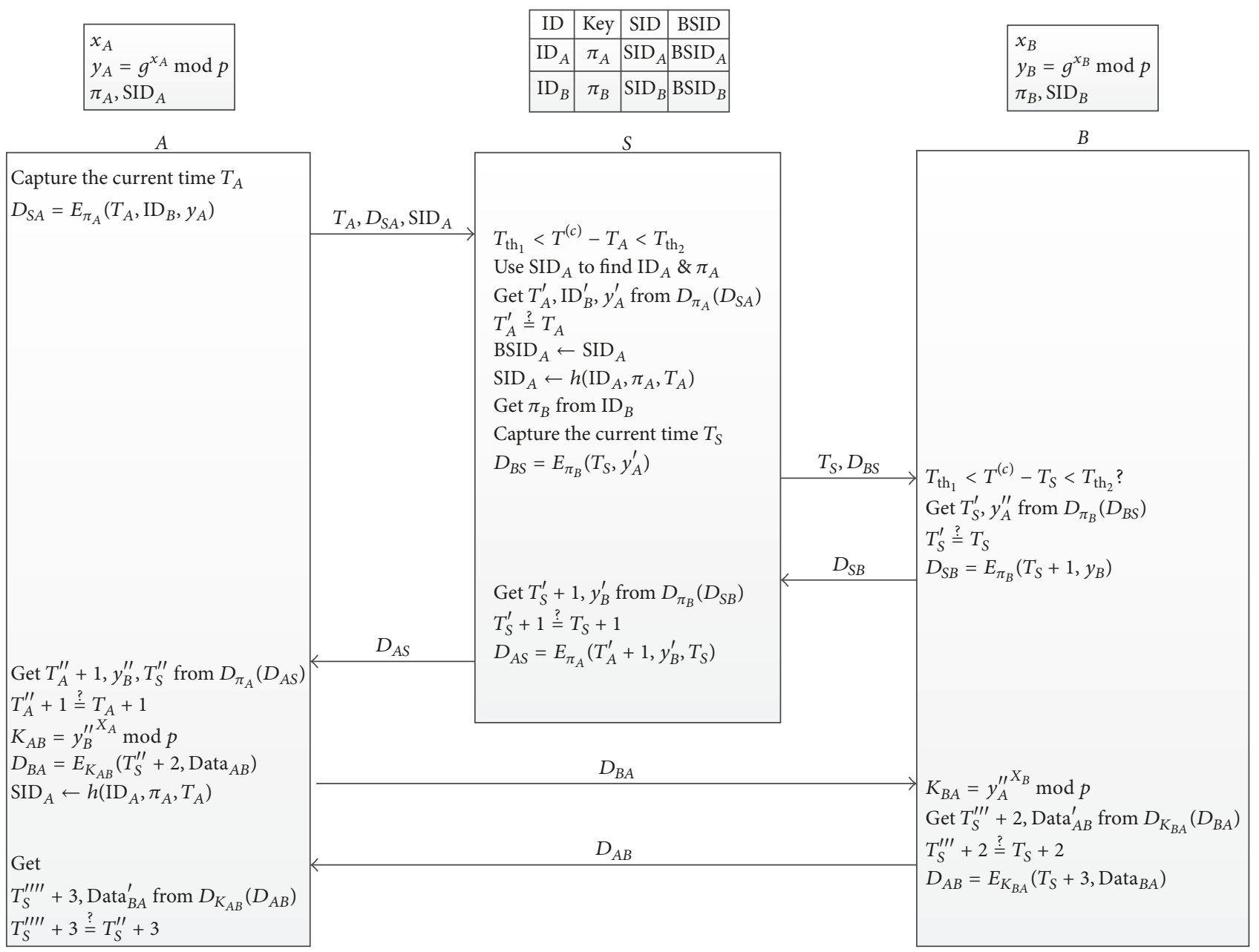

FIgURE 5: Procedure of the proposed scheme.

(3) Authenticity: any participant can authenticate other participants including the server.

(4) Medical record confidentiality: an adversary (including the server) cannot disclose any medical records.

(5) Medical record nonforgeability: an adversary cannot successfully forge electronic medical records.

(6) Resistance to asynchronous attacks: the system can process a successful authentication even if the data stored in participants' database may be asynchronous when a session cannot be normally completed.

(7) Resistance to tracking attack: an adversary cannot trace the medical institution $A$ or $B$ through the eavesdropped data.

\section{Proposed Scheme}

In this section, we propose a new three-party authentication scheme to achieve the functional requirements outlined in Definition 1 and the security goals outlined in Definition 2. The procedure of the proposed scheme is shown in Figure 5.
Definition 1 (functional requirements of our scheme). Our scheme features three roles: medical institution $A$, medical institution $B$, and authentication server $S$. Our scheme is functional if it provides that (1) $A, B$, and $S$ can authenticate each other; (2) $A$ and $B$ can obtain a common session key; (3) $A$ and $B$ can exchange electronic medical records; (4) $S$ is not required to have a public key; and (5) it is efficient to implement.

Definition 2 (security requirements of our scheme). Our scheme is secure if it achieves the following: (1) data integrity, (2) anonymity, (3) authenticity, (4) medical record confidentiality, (5) medical record nonforgeability, (6) resistance to asynchronous attacks, and (7) resistance to tracking attacks.

4.1. Initialization Phase. This phase establishes the required parameters.

(1) The system chooses one large prime number $p$.

(2) The system chooses one primitive root $g$ modulo $p$.

(3) $A$ and $B$ each chooses a random number, respectively, $x_{A}$ and $x_{B} \in_{R} Z_{p-1}^{*}$ and, respectively, computes $y_{A}=$ $g^{x_{A}} \bmod p, y_{B}=g^{x_{B}} \bmod p$. 
(4) $A$ and $B$, respectively, generate symmetric keys $\pi_{A}$ and $\pi_{B}$ and use them to register with $S$.

(5) $A$ and $B$, respectively, compute $\operatorname{SID}_{A}=h\left(\mathrm{ID}_{A}, \pi_{A}, 0\right)$ and $\operatorname{SID}_{B}=h\left(\mathrm{ID}_{B}, \pi_{B}, 0\right)$.

(6) $S$ stores $\mathrm{ID}_{X}, \pi_{X}$, and $\operatorname{SID}_{X}=h\left(\mathrm{ID}_{X}, \pi_{X}, 0\right)$, where $X=A$ or $B$.

4.2. Verification Phase. This phase presents the process of mutual authentication, key exchange, and data transmission among $A, B$, and $S$.

(1) $A \rightarrow S: T_{A}, D_{S A}, \operatorname{SID}_{A}: A$ obtains the current time $T_{A}$, computes $D_{S A}=E_{\pi_{A}}\left(T_{A}, \mathrm{ID}_{B}, y_{A}\right)$, and sends $T_{A}$, $D_{S A}$, and $\operatorname{SID}_{A}$ to $S$.

(2) $S$ authenticates $A$ : $S$ verifies whether $T_{\mathrm{th}_{1}}<T^{(c)}-$ $T_{A}<T_{\mathrm{th}_{2}}$ holds, uses $\mathrm{SID}_{A}$ to find $\mathrm{ID}_{A}$, decrypts $D_{S A}$ via key $\pi_{A}$ to obtain $T_{A}^{\prime}, \mathrm{ID}_{B}^{\prime}$, and $y_{A}^{\prime}$, and evaluates whether $T_{A}^{\prime}$ equals $T_{A}$. If it is, the identity of $A$ is authenticated.

(3) $S$ updates $\operatorname{BSID}_{A}: S$ updates $\operatorname{BSID}_{A} \leftarrow \operatorname{SID}_{A}$.

(4) $S$ updates $\operatorname{SID}_{A}: S$ updates $\operatorname{SID}_{A} \leftarrow h\left(\operatorname{ID}_{A}, \pi_{A}, T_{A}\right)$.

(5) $S \rightarrow B: T_{S}, D_{B S}: S$ obtains the current time $T_{S}$, uses $\mathrm{ID}_{B}^{\prime}$ to find $\pi_{B}$, computes $D_{B S}=E_{\pi_{B}}\left(T_{S}, y_{A}^{\prime}\right)$, and sends $T_{S}$ and $D_{B S}$ to $B$.

(6) $B$ authenticates $S$ : $B$ verifies whether $T_{\mathrm{th}_{1}}<T^{(c)}-T_{S}<$ $T_{\mathrm{th}_{2}}$ holds, decrypts $D_{B S}$ via key $\pi_{B}$ to obtain $T_{S}^{\prime}$ and $y_{A}^{\prime \prime}$, and evaluates whether $T_{S}$ equals $T_{S}^{\prime}$. If so, the identity of $S$ is authenticated.

(7) $B \rightarrow S: D_{S B}: B$ computes $D_{S B}=E_{\pi_{B}}\left(T_{S}+1, y_{B}\right)$ and sends $D_{S B}$ to $S$.

(8) $S$ authenticates $B$ : $S$ decrypts $D_{S B}$ via key $\pi_{B}$ to obtain $T_{S}^{\prime}+1$ and $y_{B}^{\prime}$ and evaluates whether $T_{S}^{\prime}+1$ equals $T_{S}+1$. If so, the identity of $B$ is authenticated.

(9) $S \rightarrow A: D_{A S}: S$ computes $D_{A S}=E_{\pi_{A}}\left(T_{A}^{\prime}+1, y_{B}^{\prime}, T_{S}\right)$ and sends $D_{A S}$ to $A$.

(10) $A$ authenticates $S$ : $A$ decrypts $D_{A S}$ via key $\pi_{A}$ to obtain $T_{A}^{\prime \prime}+1, y_{B}^{\prime \prime}$, and $T_{S}^{\prime \prime}$ and evaluates whether $T_{A}^{\prime \prime}+1$ equals $T_{A}+1$. If so, the identity of $S$ is authenticated. $A$ then computes $K_{A B}=y_{B}^{\prime \prime} X_{A} \bmod p$.

(11) $A \rightarrow B: D_{B A}: A$ computes $D_{B A}=E_{K_{A B}}\left(T_{S}^{\prime \prime}+\right.$ 2, Data $A B$ ) and sends $D_{B A}$ to $B$.

(12) $A$ updates $\operatorname{SID}_{A}: A$ updates $\operatorname{SID}_{A} \leftarrow h\left(\operatorname{ID}_{A}, \pi_{A}, T_{A}\right)$.

(13) $B$ authenticates $A: B$ computes $K_{B A}=y_{A}^{\prime \prime X_{B}} \bmod p$, decrypts $D_{B A}$ via key $K_{B A}$ to obtain $T_{S}^{\prime \prime \prime}+2$ and Data $_{A B}^{\prime}$, and evaluates whether $T_{S}^{\prime \prime \prime}+2$ equals $T_{S}+2$. If so, the identity of $A$ is authenticated, while the data Data $_{A B}^{\prime}$ is also obtained.

(14) $B \rightarrow A: D_{A B}: B$ computes $D_{A B}=E_{K_{B A}}\left(T_{S}+3\right.$, Data $_{B A}$ ) and sends $D_{A B}$ to $A$.

(15) $A$ authenticates $B$ : $A$ decrypts $D_{A B}$ via key $K_{A B}$ to obtain $T_{S}^{\prime \prime \prime \prime}+3$ and $D^{\prime \prime a t a}{ }_{B A}^{\prime}$ and evaluates whether $T_{S}^{\prime \prime \prime \prime}+3$ equals $T_{S}^{\prime \prime}+3$. If so, the identity of $B$ is authenticated, while the data Data $_{B A}^{\prime}$ is also obtained.

\section{Security Analysis}

In this section, we analyze our protocol according to the security requirements defined in Section 3.3. The proof uses security reduction similar to that used in the random oracle model [22]. In other words, based on the security goal and attacker model, we prove that "if one claimed security property of our scheme is broken then one atomic primitive is broken," where the atomic primitive means some basic cryptographic algorithm or hard mathematical problem. Therefore, our scheme provides this claimed security property since the atomic primitive is assumed to be secure.

5.1. Data Integrity. If the transmitted data $D_{U V}$ is altered, postdecryption verification will fail, thus ensuring data integrity. Theorem 4 proves the property of data integrity from Definition 3.

Definition 3 (modified symmetric encryption problem). Let $x, y, y^{\prime} \in Z, c=E_{k}(x, y)$, and $c^{\prime}=E_{k}\left(x, y^{\prime}\right)$, where $y \neq y^{\prime}$. If $c^{\prime}$ and $y^{\prime}$ can be evaluated from given $x$ and $c$, then we say the modified symmetric encryption problem is solved (the probability of solving this problem is denoted as $\operatorname{Pr}\left(c^{\prime}, y^{\prime}\right.$ । $\left.x, c)=\varepsilon_{1}\right)$.

Theorem 4 (data integrity). In our scheme, if an adversary can change $\left(\right.$ Data $\left._{A B}, D_{B A}\right)$ to (Data $\left.a_{A B}^{\prime}, D_{B A}^{\prime}\right)$ successfully, then the modified symmetric encryption problem can be solved.

Proof. In our scheme, assume an adversary tries to change $\left(\right.$ Data $_{A B}, D_{B A}$ ) to (Data ${ }_{A B}^{\prime}, D_{B A}^{\prime}$ ) from eavesdropped $T_{S}$ and $D_{B A}$. Let $\mathrm{RO}_{1}$ be a random oracle: input $T_{S}$ and $D_{B A}$ to output Data $A B$ and $D_{B A}^{\prime}$ such that $D_{K_{A B}}\left(D_{B A}^{\prime}\right)=\left(T_{S}+2\right.$, Data $\left.{ }_{A B}^{\prime}\right)$, where $D_{B A}^{\prime} \neq D_{B A}$ (i.e., $\mathrm{RO}_{1}\left(T_{S}, D_{B A}\right) \Rightarrow$ Data $_{A B}^{\prime}, D_{B A}^{\prime}$ : $D_{K_{A B}}\left(D_{B A}^{\prime}\right)=\left(T_{S}+2\right.$, Data $\left.\left.{ }_{A B}^{\prime}\right)\right)$. In Definition 3, let $k_{A B} \leftarrow k$ and Data $A B \leftarrow y$, and let $T_{S} \leftarrow x-2, D_{B A} \leftarrow c$ be input parameters of $\mathrm{RO}_{1}$ and obtain output Data ${ }_{A B}^{\prime}$ and $D_{B A}^{\prime}$. Let $y^{\prime} \leftarrow$ Data $_{A B}^{\prime}$ and $c^{\prime} \leftarrow D_{B A}^{\prime}$; then $y^{\prime}$ is evaluated. Therefore, $\operatorname{Pr}\left(\right.$ Data $\left._{A B}^{\prime}, D_{B A}^{\prime} \mid T_{S}, D_{B A}\right) \leq \operatorname{Pr}\left(c^{\prime}, y^{\prime} \mid x, c\right)=\varepsilon_{1}$, which means the modified symmetric encryption problem can be solved if $\mathrm{RO}_{1}$ exists.

5.2. Anonymity. If the attacker wants to obtain $\mathrm{ID}_{A}$ or $\mathrm{ID}_{B}$, he has to use $\operatorname{SID}_{A}$ or $D_{S A}$ to evaluate them. However, $\mathrm{ID}_{A}$ cannot be evaluated from $\mathrm{SID}_{A} \leftarrow h\left(\mathrm{ID}_{A}, \pi_{A}, T_{A}\right)$ because of the nonreversible one-way hash function. Moreover, evaluating $\mathrm{ID}_{B}$ requires decrypting $D_{S A}=E_{\pi_{A}}\left(T_{A}, \mathrm{ID}_{B}, y_{A}\right)$ using the key $\pi_{X}$, which is not obtained or evaluated through the eavesdropped data, therefore ensuring anonymity. Theorem 6 proves the property of data integrity from Definition 5.

Definition 5 (modified hash problem). Let $a, b, c \in Z$, and $d=h(a, b, c)$. If $a$ can be evaluated from given $d$ and $c$, then we say the modified hash problem is solved (the probability of solving this problem is denoted as $\left.\operatorname{Pr}(a \mid d, c)=\varepsilon_{2}\right)$.

Theorem 6 (anonymity). In our scheme, if an administrator can obtain $I D_{A}$ from $S I D_{A}$ and $T_{A}$, then the modified hash problem can be solved. 
Proof. In our scheme, assume an adversary tries to evaluate $\mathrm{ID}_{A}$ from eavesdropped $\mathrm{SID}_{A}$ and $T_{S}$. Let $\mathrm{RO}_{2}$ be a random oracle: input $T_{S}$ and $\operatorname{SID}_{A}$ to output $\mathrm{ID}_{A}$ (i.e., $\left.\mathrm{RO}_{2}\left(T_{S}, \mathrm{SID}_{A}\right) \Rightarrow \mathrm{ID}_{A}\right)$. In Definition 5, let $\pi_{A} \leftarrow b$, and let $T_{S} \leftarrow c$ and SID $_{A} \leftarrow d$ be input parameters of $\mathrm{RO}_{2}$ and obtain output $\mathrm{ID}_{A}$. Let $a \leftarrow \mathrm{ID}_{A}$; then $a$ is evaluated. Therefore, $\operatorname{Pr}\left(\mathrm{ID}_{A} \mid \mathrm{SID}_{A}, T_{S}\right) \leq \operatorname{Pr}(a \mid d, c)=\varepsilon_{2}$, which means the modified hash problem can be solved if $\mathrm{RO}_{2}$ exists.

5.3. Authenticity. Authenticating a participant requires evaluating whether $T_{X}$ is equal to $T_{X}^{\prime}$. Although an adversary can create a new $T_{X}$ or obtain $T_{X}$ through the eavesdropped data, he cannot obtain $\pi_{X}$ or $K_{U V}$ to decrypt $T_{X}$ to achieve successful authentication, therefore ensuring authenticity. Theorem 8 proves the property of data integrity from Definition 7.

Definition 7 (joint modified symmetric encryption problem). Let $a_{i}, b, c, d_{i} \in Z$ and $d_{i}=E_{k}\left(a_{i}, b, c\right)$, where $i=1,2, \ldots, n+1$. If $\left(a_{n+1}, d_{n+1}\right)$ can be evaluated from given $c$ and $\left(a_{j}, d_{j}\right)$, then we say the joint modified symmetric encryption problem is solved, where $j=1,2, \ldots, n$ (the probability of solving this problem is denoted as $\left.\operatorname{Pr}\left(a_{n+1}, d_{n+1} \mid c, a_{j}, d_{j}\right)=\varepsilon_{3}\right)$.

Theorem 8 (authenticity). In our scheme, if $D_{S A}$ and $T_{A}$ can be forged, then the joint modified symmetric encryption problem can be solved.

Proof. In our scheme, assume an adversary tries to evaluate $T_{A}{ }^{(n+1)}, D_{S A}{ }^{(n+1)}$ to forge the identity of $A$ from $y_{A}$ and eavesdropped $\left(T_{A}{ }^{(i)}, D_{S A}{ }^{(i)}\right), i=1,2, \ldots, n$. Let $\mathrm{RO}_{3}$ be a random oracle: input $T_{A}{ }^{(i)}, D_{S A}{ }^{(i)}, y_{A}$ to output $D_{S A}{ }^{(n+1)}, T_{A}{ }^{(n+1)}, i=$ $1,2, \ldots, n$ (i.e., $\left.\mathrm{RO}_{3}\left(\left(T_{A}{ }^{(i)}, D_{S A}{ }^{(i)}\right), y_{A}\right) \Rightarrow T_{A}{ }^{(n+1)}, D_{S A}{ }^{(n+1)}\right)$. In Definition 7, let $T_{A}{ }^{(i)} \leftarrow a_{i}, D_{S A}{ }^{(i)} \leftarrow d_{i}$, and $y_{A} \leftarrow c$ be input parameters of $\mathrm{RO}_{3}$ and obtain output $D_{S A}{ }^{(n+1)}, T_{A}{ }^{(n+1)}, i=1,2, \ldots, n$. Let $d_{n+1} \leftarrow D_{S A}{ }^{(n+1)}$ and $a_{n+1} \leftarrow T_{A}{ }^{(n+1)}$, and then $\left(a_{n+1}, d_{n+1}\right)$ are evaluated. Therefore, $\operatorname{Pr}\left(D_{S A}{ }^{(n+1)}, T_{A}{ }^{(n+1)} \mid D_{S A}{ }^{(i)}, T_{A}{ }^{(i)}, y_{A}\right) \leq \operatorname{Pr}\left(a_{n+1}\right.$, $\left.d_{n+1} \mid c, a_{j}, d_{j}\right)=\varepsilon_{3}$, which means the joint modified symmetric encryption problem can be solved if $\mathrm{RO}_{3}$ exists.

5.4. Medical Record Confidentiality. Obtaining medical records requires using the session key $K_{A B}$ (or $K_{B A}$ ) to decrypt $D_{B A}$ (or $\left.D_{A B}\right)$, where the session key is generated from one's own private key $x_{A}$ (or $x_{B}$ ) and the public key $y_{B}\left(\right.$ or $\left.y_{A}\right)$ of the opposite side. An attacker can neither evaluate the private keys $x_{A}$ or $x_{B}$, nor obtain the public keys $y_{B}$ or $y_{A}$ due to anonymity. Therefore, the scheme ensures medical record confidentiality. Theorem 10 proves the property of data integrity from Definition 9.

Definition 9 (second modified symmetric problem). Let $x, y \in Z$, and $c=E_{k}(x, y)$. If $y$ can be evaluated from given $x$ and $c$, then we say the second modified symmetric problem is solved (the probability of solving this problem is denoted as $\left.\operatorname{Pr}(y \mid x, c)=\varepsilon_{4}\right)$.

Theorem 10 (medical record confidentiality). In our scheme, if attacker can obtain Data ${ }_{A B}$, then the second modified symmetric problem can be solved.
Proof. In our scheme, assume an adversary tries to obtain Data $_{A B}$ from eavesdropped $T_{S}$ and $D_{B A}$. Let $\mathrm{RO}_{4}$ be a random oracle: input $T_{S}$ and $D_{B A}$ to output $D_{a t a}$ (i.e., $\left.\mathrm{RO}_{4}\left(T_{S}, D_{B A}\right) \Rightarrow \operatorname{Data}_{A B}\right)$. In Definition 9, let $T_{S} \leftarrow x$ and $D_{B A} \leftarrow c$ be input parameters of $\mathrm{RO}_{4}$ and obtain output Data $_{A B}$. Let $y \leftarrow \operatorname{Data}_{A B}$, then $y$ is evaluated. Therefore, $\operatorname{Pr}\left(\right.$ Data $\left._{A B} \mid T_{S}, D_{B A}\right) \leq \operatorname{Pr}(y \mid x, c)=\varepsilon_{4}$, which means the second modified symmetric problem can be solved if $\mathrm{RO}_{4}$ exists.

5.5. Medical Record Nonforgeability. After decrypting $D_{A B}$ (or $D_{B A}$ ), $A$ (or $B$ ) have to authenticate each other via check$\operatorname{ing} T_{S}^{\prime \prime \prime \prime \prime}+3 \stackrel{?}{=} T_{S}^{\prime \prime}+3$ (or $T_{S}^{\prime \prime \prime}+2 \stackrel{?}{=} T_{S}+2$ ). If an attacker wants to forge a medical record Data ${ }_{B A}($ or Data $A B$ ), he/she has to evaluate the session key $K_{A B}$ (or $K_{B A}$ ) to encrypt both Data ${ }_{B A}$ and $T_{S}+3$ (or both Data $_{A B}$ and $T_{S}^{\prime \prime}+2$ ). Since the attacker cannot evaluate the session key, our scheme provides medical record nonforgeability. Theorem 12 proves the property of data integrity from Definition 11.

Definition 11 (second joint modified symmetric encryption problem). Let $x_{i}, y_{i} \in Z$ and $c_{i}=E_{k}\left(x_{i}, y_{i}\right), i=1,2, \ldots, n+1$. If $c_{n+1}$ can be evaluated from given $x_{n+1}$ and $\left(x_{j}, c_{j}\right), j=$ $1,2, \ldots, n$, then we say the second joint modified symmetric encryption problem is solved (the probability of solving this problem is denoted as $\left.\operatorname{Pr}\left(c_{n+1} \mid\left(x_{j}, c_{j}\right), x_{n+1}\right)=\varepsilon_{5}\right)$.

Theorem 12 (medical record nonforgeability). In our scheme, if $D_{B A}$ can be modified successfully, then the second joint modified symmetric encryption problem can be solved.

Proof. In our scheme, assume an adversary tries to forge $D_{B A}^{(n+1)}$ from $T_{S}^{(n+1)}$ and eavesdropped $\left(T_{S}^{(i)}, D_{B A}^{(i)}\right), i=1,2$, $\ldots, n$. Let $\mathrm{RO}_{5}$ be a random oracle: input $T_{S}^{(n+1)}$ and $\left(T_{S}^{(i)}, D_{B A}^{(i)}\right)$ to output $D_{B A}^{(n+1)}$ (i.e., $\mathrm{RO}_{5}\left(\left(T_{S}^{(i)}, D_{B A}^{(i)}\right), T_{S}^{(n+1)}\right) \Rightarrow$ $\left.D_{B A}^{(n+1)}\right)$. In Definition 11, let $T_{S}^{(i)} \leftarrow x_{i}-2, D_{B A}^{(i)} \leftarrow c_{i}$ and $T_{S}^{(n+1)} \leftarrow x_{n+1}-2$ be input parameters of $\mathrm{RO}_{5}$ and obtain output $D_{B A}^{(n+1)}$. Let $c_{n+1} \leftarrow D_{B A}^{(n+1)}$, then $c_{n+1}$ is evaluated. Therefore, $\operatorname{Pr}\left(D_{B A}^{(n+1)} \mid\left(T_{S}^{(i)}, D_{B A}^{(i)}\right), T_{S}^{(n+1)}\right) \leq \operatorname{Pr}\left(c_{n+1} \mid\left(x_{i}, c_{i}\right)\right.$, $\left.x_{n+1}\right)=\varepsilon_{5}$, which means the second joint modified symmetric encryption problem can be solved if $\mathrm{RO}_{5}$ exists.

5.6. Resistance to Asynchronous Attacks. If an attacker wants to block a communication to make an asynchronous attack, he/she can use the following methods to cause $A$ and $S$ to update $\operatorname{SID}_{A}$ asynchronously, causing the system fail in the future.

(A) Blocking $A \rightarrow S: T_{A}, D_{S A}$, $\operatorname{SID}_{A}$ : if $S$ does not receive the data sent from $A, S$ does not update $\operatorname{SID}_{A}$ and $\mathrm{BSID}_{A}$, nor does $A$ update $\mathrm{SID}_{A}$. Therefore, an asynchronous attack based on this blocking method will fail.

(B) Blocking $S \rightarrow A: D_{A S}$ : if $A$ does not receive the data sent from $S, A$ will not update $\operatorname{SID}_{A}$. In the next authentication session, $S$ will use $\mathrm{BSID}_{A}$ to determine the information of $\mathrm{ID}_{A}$. Therefore, this blocking method is resisted. 
TABLE 1: Comparison of computation and communication costs with verifier-based 3PAKA schemes without server public keys.

\begin{tabular}{|c|c|c|c|c|c|c|c|c|c|c|c|c|c|c|c|}
\hline & \multicolumn{3}{|c|}{$\begin{array}{c}\text { Lee et al.s } \\
\text { scheme [8] }\end{array}$} & \multicolumn{3}{|c|}{$\begin{array}{l}\text { Wang-Mo's } \\
\text { scheme [9] }\end{array}$} & \multicolumn{3}{|c|}{$\begin{array}{l}\text { Kwon et al's } \\
\text { scheme [10] }\end{array}$} & \multicolumn{3}{|c|}{$\begin{array}{l}\text { Lin-Lee's } \\
\text { scheme [7] }\end{array}$} & \multicolumn{3}{|c|}{ Our scheme } \\
\hline & $A$ & $B$ & $S$ & $A$ & $B$ & $S$ & $A$ & $B$ & $S$ & $A$ & $B$ & $S$ & $A$ & $B$ & $S$ \\
\hline Exponentiation & 3 & 4 & 4 & 4 & 4 & 6 & 4 & 4 & 10 & 4 & 4 & 6 & 1 & 1 & 0 \\
\hline $\mathrm{X}$-or & 1 & 1 & 2 & 1 & 1 & 2 & 0 & 0 & 0 & 1 & 1 & 2 & 0 & 0 & 0 \\
\hline Multip./division & 0 & 0 & 0 & 0 & 0 & 0 & 3 & 3 & 4 & 0 & 0 & 0 & 0 & 0 & 0 \\
\hline Random number & 1 & 1 & 2 & 1 & 1 & 2 & 1 & 1 & 4 & 1 & 1 & 2 & 0 & 0 & 0 \\
\hline Hash & 3 & 3 & 4 & 3 & 3 & 4 & 3 & 3 & 4 & 4 & 4 & 4 & 1 & 0 & 1 \\
\hline Encryption/decryption & 0 & 0 & 0 & 0 & 0 & 0 & 0 & 0 & 0 & 0 & 0 & 0 & 4 & 4 & 2 \\
\hline Transmission messages & 3 & 6 & 4 & 3 & 6 & 4 & 3 & 10 & 3 & 6 & 7 & 4 & 4 & 2 & 3 \\
\hline Transmission rounds & 2 & 3 & 4 & 2 & 3 & 4 & 2 & 4 & 2 & 4 & 4 & 4 & 2 & 2 & 2 \\
\hline
\end{tabular}

TABLE 2: Security comparison with verifier-based 3PAKA schemes w/o server public keys.

\begin{tabular}{|c|c|c|c|c|c|}
\hline Property & $\begin{array}{c}\text { Lee et al.s } \\
\text { scheme [8] }\end{array}$ & $\begin{array}{l}\text { Wang-Mo's } \\
\text { scheme [9] }\end{array}$ & $\begin{array}{l}\text { Kwon et al. 's } \\
\text { scheme [10] }\end{array}$ & $\begin{array}{l}\text { Lin-Lee's } \\
\text { scheme [7] }\end{array}$ & Our scheme \\
\hline Resistance to password guessing attacks & $r$ & $\checkmark$ & $\checkmark$ & $r$ & $\checkmark$ \\
\hline Resistance to verifier stolen attacks & & $\checkmark$ & $\checkmark$ & $\checkmark$ & $\checkmark$ \\
\hline Key confirmation & & & & $\checkmark$ & $\checkmark$ \\
\hline Resistance to impersonation attacks & & & & $\checkmark$ & $\checkmark$ \\
\hline Anonymity & & & & & $\checkmark$ \\
\hline Resistance to tracking attacks & & & & & $\checkmark$ \\
\hline Efficiency of implementation & & & & & $\checkmark$ \\
\hline
\end{tabular}

(C) Blocking $S \rightarrow B$ or $B \rightarrow S$ : blocking $S \rightarrow B$ or $B \rightarrow S$ has the same result as blocking $S \rightarrow A: D_{A S}$ which prevents asynchronous attacks.

(D) Blocking $A \rightarrow B$ or $B \rightarrow A$ : blocking $A \rightarrow B$ or $B \rightarrow A$ does not affect any update of $\operatorname{SID}_{A}$.

5.7. Resistance to Tracking Attack. Since $\mathrm{SID}_{X} \leftarrow h\left(\mathrm{ID}_{X}\right.$, $\left.\pi_{X}, T_{X}\right)$ changes in each round and the relationship between the previous and current $\operatorname{SID}_{X}$ and $T_{X}$ cannot be found, where $X=A$ or $B$, the scheme is resistant to tracking attacks. Theorem 14 proves the property of data integrity from Definition 13.

Definition 13 (modified hash problem). Let $a_{i}, b_{i}, c_{i} \in Z, d_{i}=$ $h\left(a_{i}, b_{i}, c_{i}\right), i=1,2$. If Equal $\left(a_{1}, a_{2}\right)$ can be evaluated from given $d_{1}, d_{2}, c_{1}$, and $c_{2}$, then we say the modified hash problem is solved, where $\operatorname{Equal}(x, y)=\{1$, if $x=y$; 0 , otherwise $\}$ (the probability of solving this problem is denoted as $\left.\operatorname{Pr}\left(\operatorname{Equal}\left(a_{1}, a_{2}\right) \mid d_{1}, d_{2}, c_{1}, c_{2}\right)=\varepsilon_{6}\right)$.

Theorem 14 (resistance to tracking attack). In our scheme, if attacker can evaluate Equal $\left(I D_{A}{ }^{(i)}, I D_{A}{ }^{(j)}\right)$, then the modified hash problem can be solved.

Proof. In our scheme, assume an adversary tries to evaluate $\operatorname{Equal}\left(\mathrm{ID}_{A}{ }^{(i)}, \mathrm{ID}_{A}{ }^{(j)}\right)$ to track user $A$ from eavesdropped $\operatorname{SID}_{A}{ }^{(i)}, \operatorname{SID}_{A}{ }^{(j)}, T_{A}{ }^{(i)}$, and $T_{A}{ }^{(j)}$. Let $\mathrm{RO}_{6}$ be a random oracle: input $\operatorname{SID}_{A}{ }^{(i)}, \operatorname{SID}_{A}{ }^{(j)}, T_{A}{ }^{(i)}$, and $T_{A}{ }^{(j)}$ to output Equal $\left(\operatorname{ID}_{A}{ }^{(i)}, \operatorname{ID}_{A}{ }^{(j)}\right)$ (i.e., $\operatorname{RO}_{6}\left(\operatorname{SID}_{A}{ }^{(i)}, \operatorname{SID}_{A}{ }^{(j)}\right.$, $\left.\left.T_{A}{ }^{(i)}, T_{A}{ }^{(j)}\right) \Rightarrow \operatorname{Equal}\left(\operatorname{ID}_{A}{ }^{(i)}, \operatorname{ID}_{A}{ }^{(j)}\right)\right)$. In Definition 13, let
$\operatorname{SID}_{A}{ }^{(i)} \leftarrow d_{i}, \operatorname{SID}_{A}{ }^{(j)} \leftarrow d_{j}, T_{A}{ }^{(i)} \leftarrow c_{i}$, and $T_{A}{ }^{(j)} \leftarrow c_{j}$ be input parameters of $\mathrm{RO}_{6}$ and obtain output $\operatorname{Equal}\left(\mathrm{ID}_{A}{ }^{(i)}, \mathrm{ID}_{A}{ }^{(j)}\right)$. Let $\operatorname{Equal}\left(a_{1}, a_{2}\right) \leftarrow \operatorname{Equal}\left(\mathrm{ID}_{A}{ }^{(i)}\right.$, $\left.\operatorname{ID}_{A}{ }^{(j)}\right)$, then $\operatorname{Equal}\left(a_{1}, a_{2}\right)$ is evaluated. Therefore, $\operatorname{Pr}\left(\operatorname{Equal}\left(\mathrm{ID}_{A}{ }^{(i)}, \operatorname{ID}_{A}{ }^{(j)}\right) \quad \mid \operatorname{SID}_{A}{ }^{(i)}, \operatorname{SID}_{A}{ }^{(j)}, T_{A}{ }^{(i)}, T_{A}{ }^{(j)}\right) \leq$ $\operatorname{Pr}\left(\operatorname{Equal}\left(a_{1}, a_{2}\right) \quad \mid d_{1}, d_{2}, c_{1}, c_{2}\right)=\varepsilon_{6}$, which means the modified hash problem can be solved if $\mathrm{RO}_{6}$ exists.

\section{Comparison}

This section compares the performance of our proposed method with previous verifier-based three-party authentication and key agreement (3PAKA) protocols without server public keys in terms of two aspects: computation and communication loadings (as shown in Table 1) and system properties (as shown in Table 2). From Table 1, our scheme is superior to previous schemes in terms of computation and communication performance. Table 2 shows that, unlike other schemes, our proposed scheme provides anonymity and resistance to tracking attacks. Therefore, our scheme is superior to previous schemes in terms of security.

Moreover, in Tables 3 and 4, we compare our proposed method with previous RSA-based 3PAKA schemes in terms of computation and communication loadings and system properties. Our protocol is a secure scheme providing 3PAKA and data exchange in medical environment, and the computation and communication loadings for providing only 3PAKA (without data exchange) in our scheme are different (lesser). In Table 4, Chang et al.s [11] and Tso's [12] schemes do not support mutual authentication, and Deebak et al's scheme 


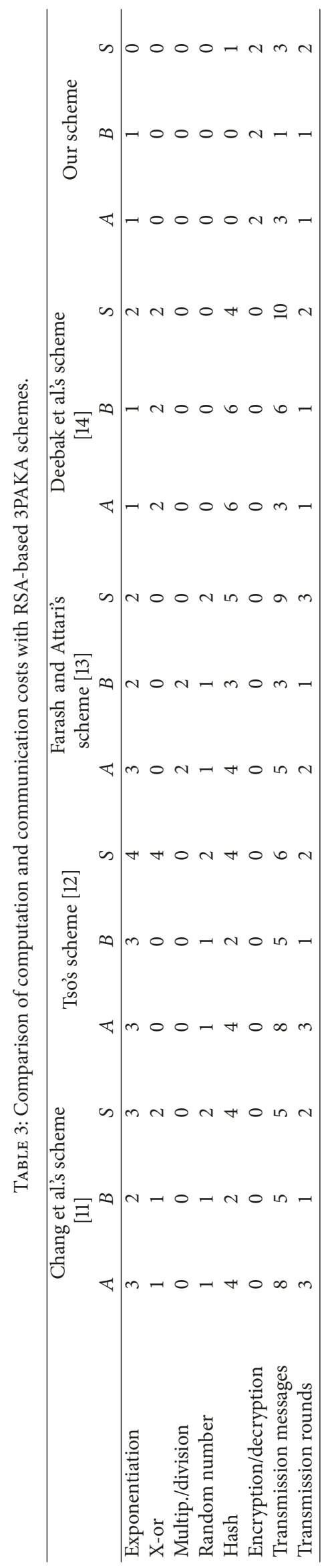


TABLE 4: Security comparison with RSA-based 3PAKA schemes.

\begin{tabular}{lccccc}
\hline Property & $\begin{array}{c}\text { Chang et al's } \\
\text { scheme [11] }\end{array}$ & Tso's scheme [12] & $\begin{array}{c}\text { Farash and Attari's } \\
\text { scheme [13] }\end{array}$ & $\begin{array}{c}\text { Deebak et al's } \\
\text { scheme [14] }\end{array}$ & $\begin{array}{c}\text { Our scheme } \\
\text { Resistance to password guessing attacks }\end{array}$ \\
Resistance to verifier stolen attacks & N/A & $\checkmark$ & $\checkmark$ & $\checkmark$ & $\checkmark$ \\
Key confirmation & $\checkmark$ & N/A & $\checkmark$ & $\checkmark$ & $\checkmark$ \\
Resistance to impersonation attacks & $\checkmark$ & $\checkmark$ & $\checkmark$ & $\checkmark$ & $\checkmark$ \\
Anonymity & & & & $\checkmark$ \\
Resistance to tracking attacks & & & & $\checkmark$ \\
Efficiency of implementation & & & & $\checkmark$ \\
\hline
\end{tabular}

TABLE 5: Comparison of computation and communication costs with other 3PAKA schemes.

\begin{tabular}{|c|c|c|c|c|c|c|c|c|c|c|c|c|}
\hline & \multicolumn{3}{|c|}{$\begin{array}{c}\text { Chen et at.'s scheme } \\
\text { [15] }\end{array}$} & \multicolumn{3}{|c|}{$\begin{array}{l}\text { Wang et al's scheme } \\
{[16]}\end{array}$} & \multicolumn{3}{|c|}{$\begin{array}{c}\text { Farash et al's scheme } \\
{[17]}\end{array}$} & \multicolumn{3}{|c|}{ Our scheme } \\
\hline & $A$ & $B$ & $S$ & $A$ & $B$ & $S$ & $A$ & $B$ & $S$ & $A$ & $B$ & $S$ \\
\hline Exponentiation & 3 & 3 & 2 & 4 & 4 & 6 & 2 & 3 & 2 & 1 & 1 & 0 \\
\hline $\mathrm{X}$-or & 0 & 0 & 0 & 1 & 1 & 2 & 1 & 2 & 3 & 0 & 0 & 0 \\
\hline Multip./division & 0 & 0 & 0 & 0 & 0 & 0 & 0 & 0 & 0 & 0 & 0 & 0 \\
\hline Random number & 1 & 1 & 0 & 1 & 1 & 1 & 1 & 1 & 0 & 0 & 0 & 0 \\
\hline Hash & 2 & 2 & 2 & 3 & 3 & 4 & 1 & 2 & 2 & 0 & 0 & 1 \\
\hline Encryption/decryption & 0 & 0 & 0 & 2 & 2 & 3 & 0 & 0 & 0 & 2 & 2 & 2 \\
\hline Transmission messages & 5 & 11 & 2 & 3 & 8 & 1 & 4 & 10 & 5 & 3 & 1 & 3 \\
\hline Transmission rounds & 2 & 2 & 1 & 2 & 1 & 4 & 1 & 2 & 1 & 1 & 1 & 2 \\
\hline
\end{tabular}

TABLE 6: Security comparison with other 3PAKA schemes.

\begin{tabular}{lcccc}
\hline Property & Chen et at.'s scheme [15] & Wang et al.s scheme [16] & Farash et at.s scheme [17] & Our scheme \\
\hline Resistance to password guessing attacks & $\checkmark$ & $\checkmark$ & $\checkmark$ & $\checkmark$ \\
Resistance to verifier stolen attacks & $\checkmark$ & $\checkmark$ & N/A & $\checkmark$ \\
Key confirmation & $\checkmark$ & $\checkmark$ & $\checkmark$ & $\checkmark$ \\
Resistance to impersonation attacks & $\checkmark$ & & $\checkmark$ \\
Anonymity & & & $\checkmark$ \\
Resistance to tracking attacks & & & $\checkmark$ \\
Efficiency of implementation & & $\checkmark$ & $\checkmark$ \\
\hline
\end{tabular}

[14] requires clients to logon the system successfully before starting the 3PAKA protocol in each time. In the logon phase of Deebak et al.s scheme [14], each IP multimedia system (IMS) client enters his/her credentials into the registration form to avail the multimedia services, like video, voice, and data, and subsequently, the IMS server executes some steps to verify whether the client authorization is success or not. Moreover, each client has to perform two hash and two XOR functions in the logon phase, and the server has to perform four inverse computations in the authentication phase. Furthermore, we also compare our proposed method with other recently published 3PAKA schemes in terms of computation and communication loadings (Table 5 ) and system properties (Table 6). The schemes $[15,16]$ are smart card based schemes and some of their security and efficiency are based on smart cards. The properties "resistance to verifier-stolen attacks" of the schemes [11-13, 17] are all "N/A" because they are password-based schemes without verifiers. From Tables 3-6, our scheme is superior to previous RSA-based and other recently published 3PAKA schemes in terms of security and computation and communication performance.

\section{Implementation}

This section presents the implementation of our scheme in mobile phones (Android 4.1) and PCs (Windows 7). The mobile system is implemented on Google Nexus $S$ with a $1 \mathrm{GHz}$ processor and $512 \mathrm{MB}$ RAM. The PC implementation used Windows 7 Professional with an Intel (R) core (TM) CPU i5-650@3.2 Hz and 4 GB RAM. The server $S$ (Server 1) and the user $B$ (Server 2) are implemented on Windows 7, while the user $A$ (Telecare) is implemented on Android. The hash function used is SHA-256 [23] and the symmetric encryption algorithm is AES [24]. Figure 6 shows the scheme flow in terms of data transmission and Figure 7 shows the data transfer renderings. 


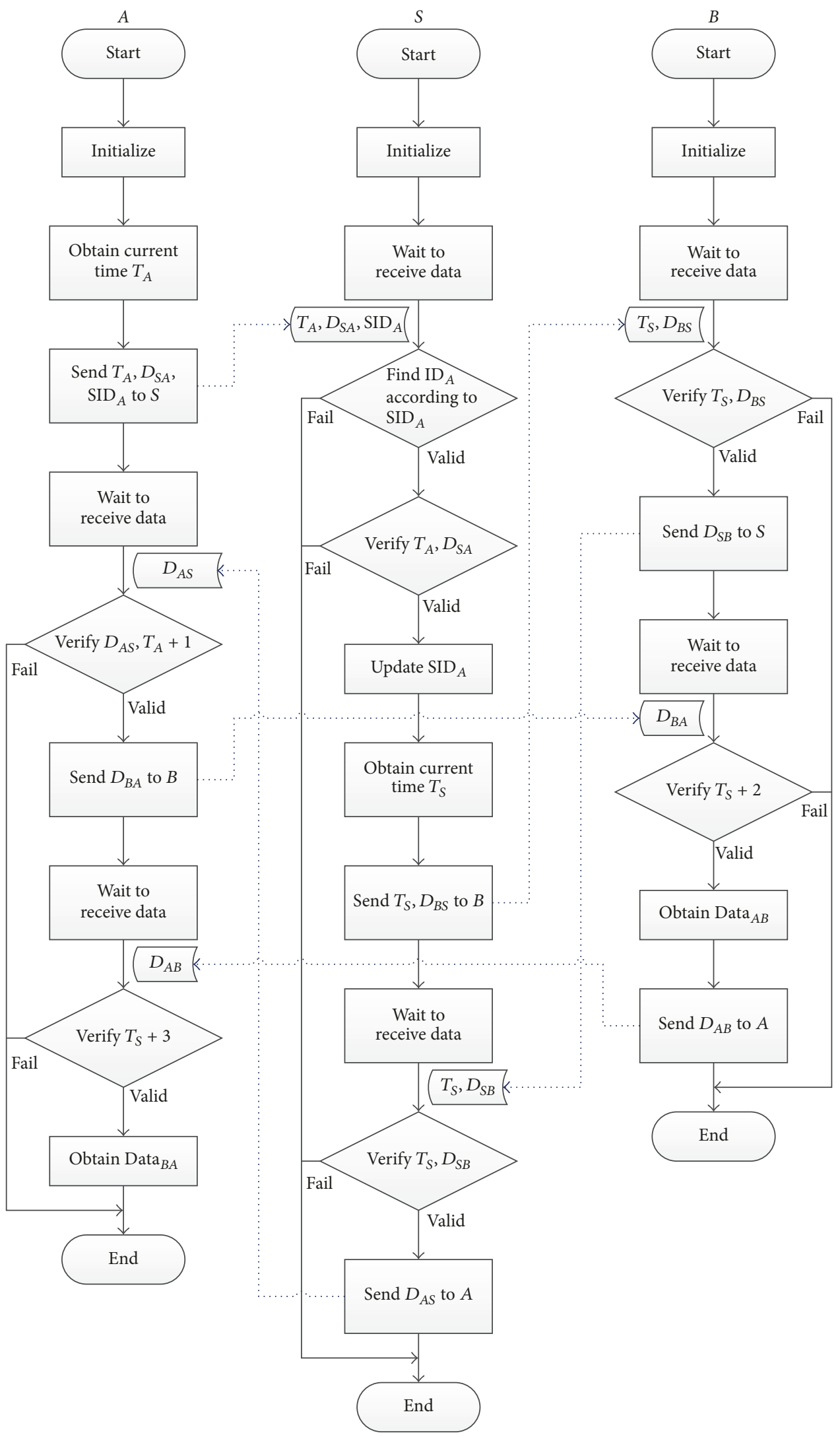

FIgURE 6: Application flowchart. 


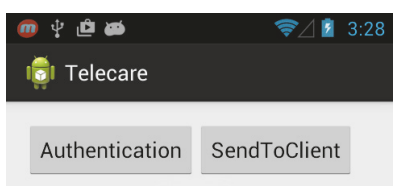

(a) Initial (on the end $A$ )

\section{Server 1}

\section{3:27:57 Server: waiting for connection...}

(b) Initial (on the end $S$ )

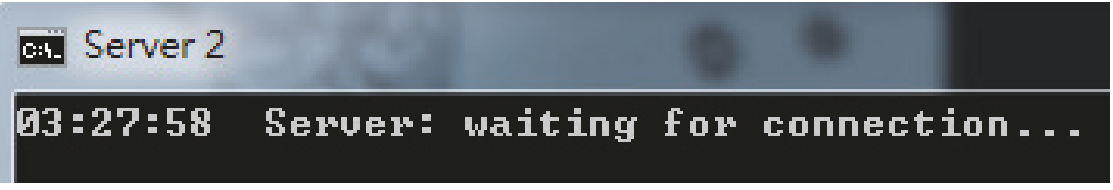

(c) Initial (on the end $B$ )

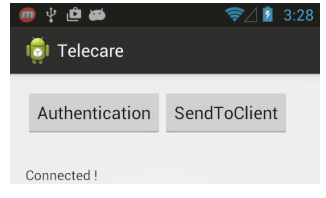

(d) Connected with $S$ (on the end $A$ )

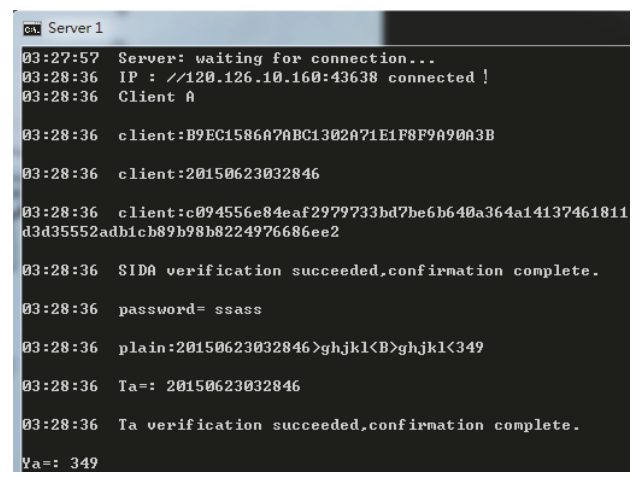

(e) Verification data received from $A$ (on the end $S$ )

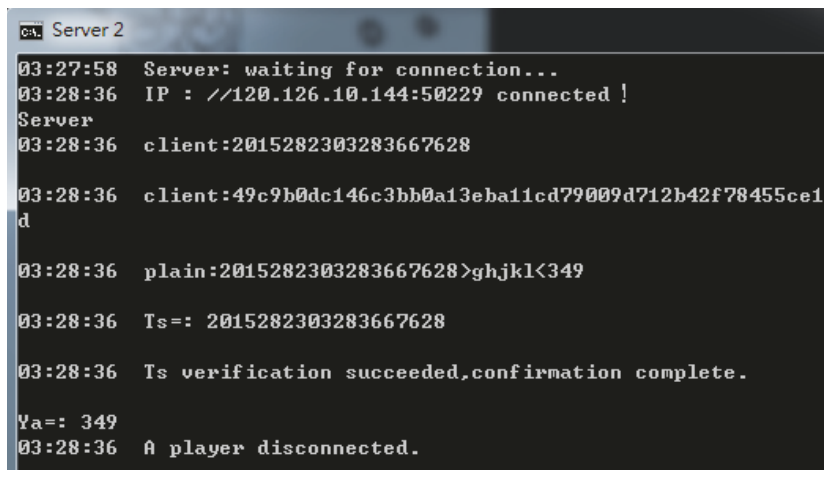

(f) Verification data received from $S$ (on the end $B$ )

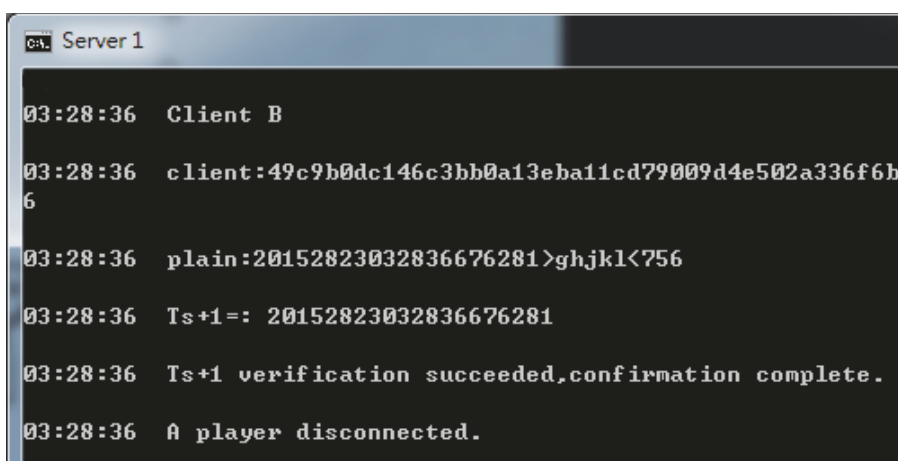

(g) Verification data received from $B$ (on the end $S$ )

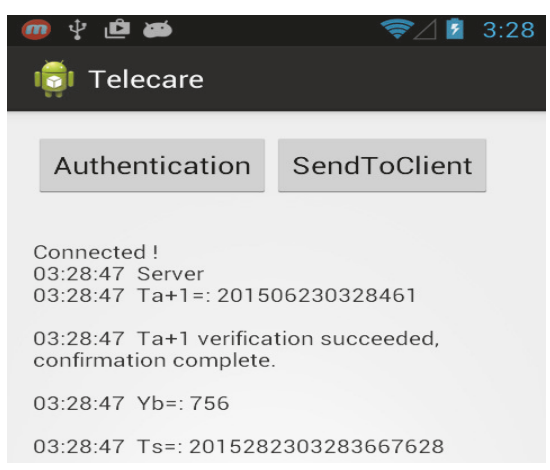

(h) Verification data received from $S$ (on the end A)

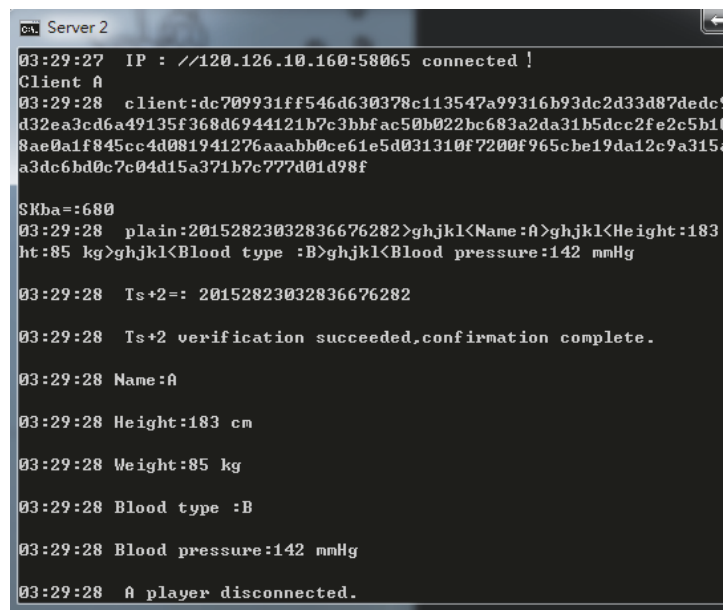

(i) (Verification) data received from $A$ (on the end $B$ )

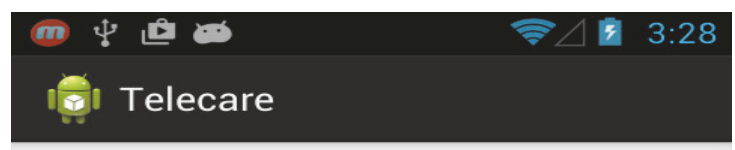
Authentication

\section{SendToClient}
Connected!
03:28:47 Server
03:28:47 Ta+1=: 201506230328461
03:28:47 $\mathrm{Ta}+1$ verification succeeded, confirmation complete.
03:28:47 Yb=: 756
03:28:47 Ts=: 2015282303283667628

(j) (Verification) data received from $B$ (on the end $A$ )

FIGURE 7: Data transfer renderings. 
In our implementation, we assume that the system times of the server $S$ and the users $A$ and $B$ are synchronized. However, their system times are difficult to be synchronized. Fortunately, the experience in implementation shows that the system time difference between the server and the users is within miniseconds. By assuming the maximum system time difference between the clients and the server is 1000 miniseconds and the values $T^{(c)}-T_{A}$ and $T^{(c)}-T_{S}$ are between $10 \mathrm{~ms}$ and $30 \mathrm{~ms}$, we suggest to set $T_{\mathrm{th}_{1}}$ and $T_{\mathrm{th}_{2}}$ to $-990 \mathrm{~ms}$ and $1030 \mathrm{~ms}$, respectively (in real situation, the values $T^{(c)}-T_{A}$ and $T^{(c)}-T_{S}$ are suggested to be measured again for much accuracy).

\section{Conclusion}

In this paper, we review Lin-Lee's protocol and demonstrate its lack of anonymity and resistance to tracking attacks. We propose an enhanced three-party authentication scheme for use in telecare medicine information systems, which provides high standards of security issues and performance. The proposed scheme does not need server public keys, reduces computation costs, and resolves two significant security issues (anonymity and resistance to tracking attacks). Comparisons with other approaches show the proposed scheme provides improved security while incurring computational, communication, and transaction costs comparable to other methods.

\section{Notations}

$\begin{array}{ll}A, B: & \text { Medical institutions } \\ S: & \text { Server } \\ p: & \text { A large prime } \\ g: & \text { Primitive root modulo } p \\ \mathrm{ID}_{X}: & \text { ID of } X \\ \text { SID }_{X}: & \text { Pseudo ID of } X \\ \mathrm{BSID}_{X}: & \text { The last pseudo ID of } X \\ T_{X}: & \text { The time of } X \\ T^{(c)}: & \text { The current time } \\ T_{\mathrm{th}}: & \text { The ith time threshold } \\ x_{A}, x_{B}: & \text { Private keys of } A \text { and } B \\ y_{A}, y_{B}: & \text { Public keys of } A \text { and } B \\ \pi_{X}: & \text { A symmetric key of } X \\ K_{A B}, K_{B A}: & \text { Section keys between } A \text { and } B \\ D_{U V}: & \text { Encrypted data from } V \text { to } U \\ h(\cdot): & \text { A one-way hash function } \\ E_{K}(\cdot): & \text { A symmetric encryption using key } K \\ D_{K}(\cdot): & \text { A symmetric decryption using key } K .\end{array}$

\section{Disclosure}

This article does not contain any studies with human participants or animals performed by any of the authors.

\section{Conflicts of Interest}

The authors declare that they have no conflicts of interest.

\section{Acknowledgments}

This work is partially supported by the Ministry of Science and Technology under Grant MOST 104-2221-E-182-012 and by the CGMH project under Grant BMRPB46.

\section{References}

[1] Y.-C. Chen, G. Horng, Y.-J. Lin, and K.-C. Chen, "Privacy preserving index for encrypted electronic medical records," Journal of Medical Systems, vol. 37, no. 6, article no. 9992, 2013.

[2] A. AlJarullah and S. El-Masri, "A Novel System Architecture for the National Integration of Electronic Health Records: A SemiCentralized Approach," Journal of Medical Systems, vol. 37, no. 4, 2013.

[3] L. Liu, J. Lai, R. H. Deng, and Y. Li, "Ciphertext-policy attributebased encryption with partially hidden access structure and its application to privacy-preserving electronic medical record system in cloud environment," Security and Communication Networks, vol. 9, no. 18, pp. 4897-4913, 2016.

[4] M. Wazid, A. K. Das, S. Kumari, X. Li, and F. Wu, "Design of an efficient and provably secure anonymity preserving three-factor user authentication and key agreement scheme for TMIS," Security and Communication Networks, vol. 9, no. 13, pp. 19832001, 2016.

[5] J. Noh, S. Lee, J. Park, S. Shin, and B. B. Kang, "Vulnerabilities of network OS and mitigation with state-based permission system," Security and Communication Networks, vol. 9, no. 13, pp. 1971-1982, 2016.

[6] M. Benssalah, M. Djeddou, and K. Drouiche, "Dual cooperative RFID-telecare medicine information system authentication protocol for healthcare environments," Security and Communication Networks, vol. 9, no. 18, pp. 4924-4948, 2016.

[7] T.-H. Lin and T.-F. Lee, "Secure verifier-based three-party authentication schemes without server public keys for data exchange in telecare medicine information systems," Journal of Medical Systems, vol. 38, no. 5, 2014.

[8] S.-W. Lee, H.-S. Kim, and K.-Y. Yoo, "Efficient verifier-based key agreement protocol for three parties without server's public key," Applied Mathematics and Computation, vol. 167, no. 2, pp. 996-1003, 2005.

[9] R.-C. Wang and K.-R. Mo, "Security enhancement on efficient verifier-based key agreement protocol for three parties without server's public key," International Mathematical Forum. Journal for Theory and Applications, vol. 1, no. 17-20, pp. 965-971, 2006.

[10] J. O. Kwon, I. R. Jeong, K. Sakurai, and D. H. Lee, "Efficient verifier-based password-authenticated key exchange in the three-party setting," Computer Standards \& Interfaces, vol. 29, no. 5, pp. 513-520, 2007.

[11] T.-Y. Chang, M.-S. Hwang, and W.-P. Yang, "A communicationefficient three-party password authenticated key exchange protocol," Information Sciences, vol. 181, no. 1, pp. 217-226, 2011.

[12] R. Tso, "Security analysis and improvements of a communication-efficient three-party password authenticated key exchange protocol," The Journal of Supercomputing, vol. 66, no. 2, pp. 863874, 2013.

[13] M. S. Farash and M. A. Attari, "An efficient and provably secure three-party password-based authenticated key exchange protocol based on Chebyshev chaotic maps," Nonlinear Dynamics, vol. 77, no. 1-2, pp. 399-411, 2014. 
[14] B. D. Deebak, R. Muthaiah, K. Thenmozhi, and P. I. Swaminathan, "Analyzing three-party authentication and key agreement protocol for real time IP multimedia server-client systems," Multimedia Tools and Applications, vol. 75, no. 10, pp. 5795-5817, 2016.

[15] C. Chen, L. Xu, W. Fang, and T. Wu, "A Three-Party Password Authenticated Key Exchange Protocol Resistant to Stolen Smart Card Attacks," in Proceedings of the 12th International Conference on Intelligent Information Hiding and Multimedia Signal Processing, vol. 1 of Advances in Intelligent Information Hiding and Multimedia Signal Processing, pp. 331-336.

[16] Q. Wang, O. Ruan, and Z. Wang, "Security Analysis and Improvements of Three-Party Password-Based Authenticated Key Exchange Protocol," in Proceedings of the International Conference on Emerging Internetworking, Data and Web Technologies, pp. 497-508, 2017.

[17] M. S. Farash, M. A. Attari, and S. Kumari, "Cryptanalysis and improvement of a three-party password-based authenticated key exchange protocol with user anonymity using extended chaotic maps," International Journal of Communication Systems, vol. 30, no. 1, 2017.

[18] R. Amin and G. P. Biswas, "A Novel User Authentication and Key Agreement Protocol for Accessing Multi-Medical Server Usable in TMIS," Journal of Medical Systems, vol. 39, no. 3, 2015.

[19] D. Mishra, A. K. Das, A. Chaturvedi, and S. Mukhopadhyay, "A secure password-based authentication and key agreement scheme using smart cards," Journal of Information Security and Applications, vol. 23, pp. 28-43, 2015.

[20] S.-Y. Chiou, Z. Ying, and J. Liu, "Improvement of a Privacy Authentication Scheme Based on Cloud for Medical Environment," Journal of Medical Systems, vol. 40, no. 4, article no. 101, pp. 1-15, 2016.

[21] S.-Y. Chiou, "Common friends discovery for multiple parties with friendship ownership and replay-attack resistance in mobile social networks," Wireless Networks, pp. 1-15, 2016.

[22] M. Bellare and P. Rogaway, "Random oracles are practical: A paradigm for designing efficient protocols," in Proceedings of the 1st ACM conference on Computer and communications security, pp. 62-73, 1993.

[23] SHA-256, http://www.cnblogs.com/elaron/archive/2013/04/09/ 3010375.html.

[24] AES, http://blog.csdn.net/hbcuil984/article/details/5201247. 


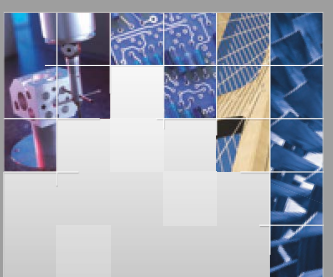

\section{Enfincering}
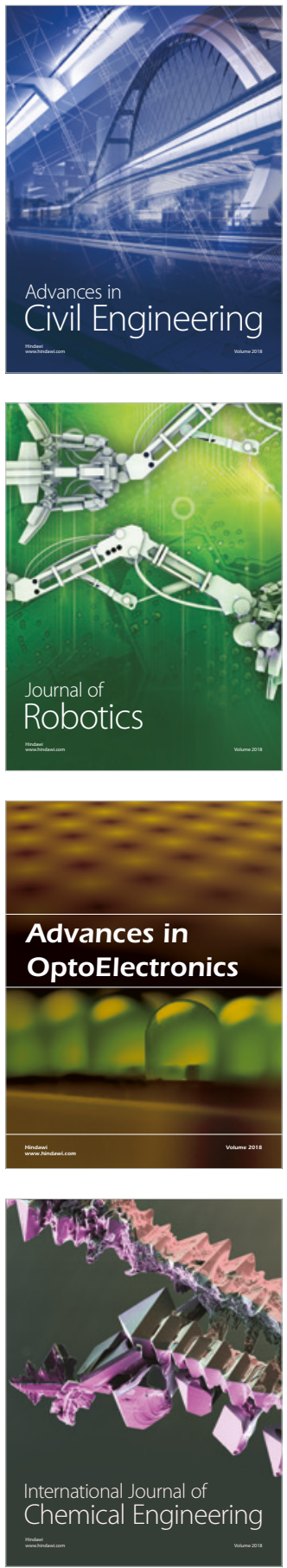

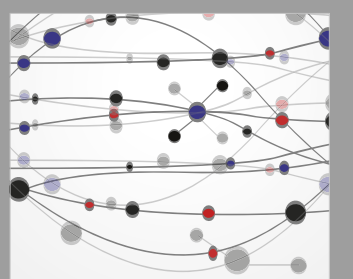

\section{Rotating \\ Machinery}

The Scientific World Journal

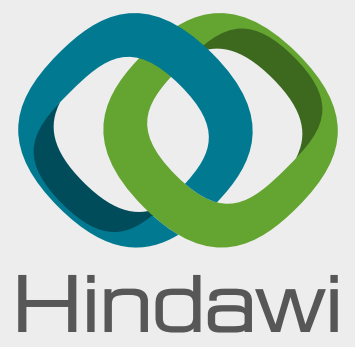

Submit your manuscripts at

www.hindawi.com
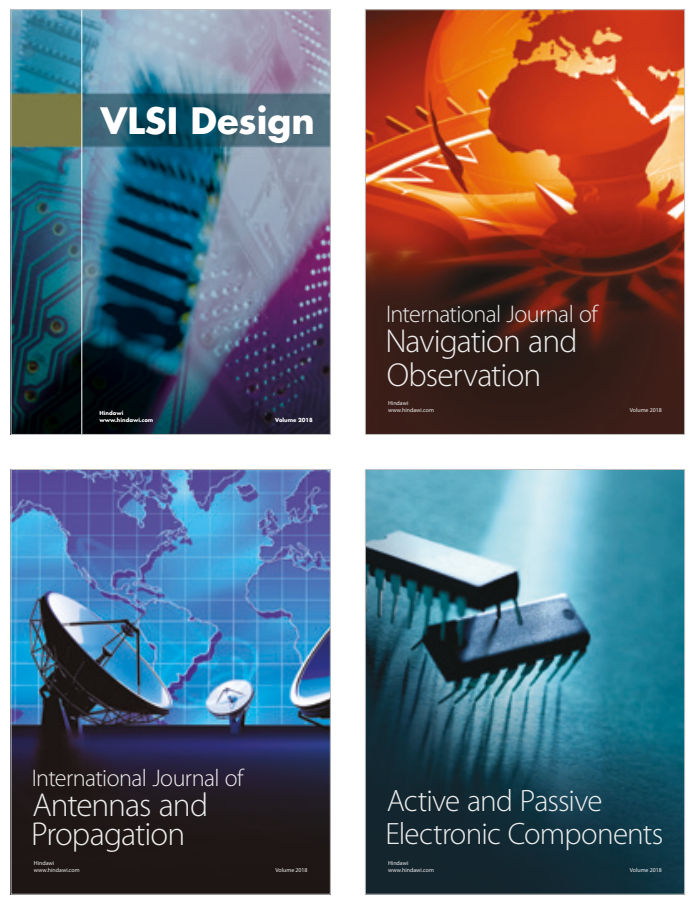
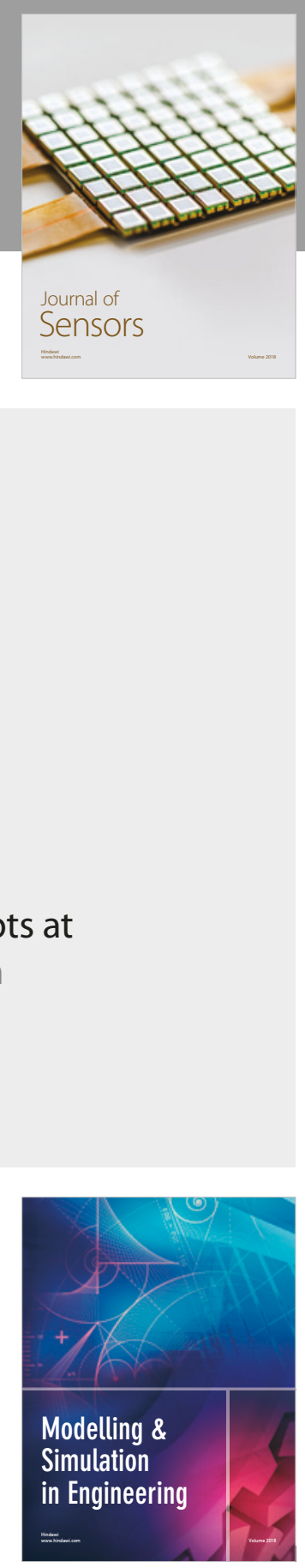

\section{Advances \\ Multimedia}
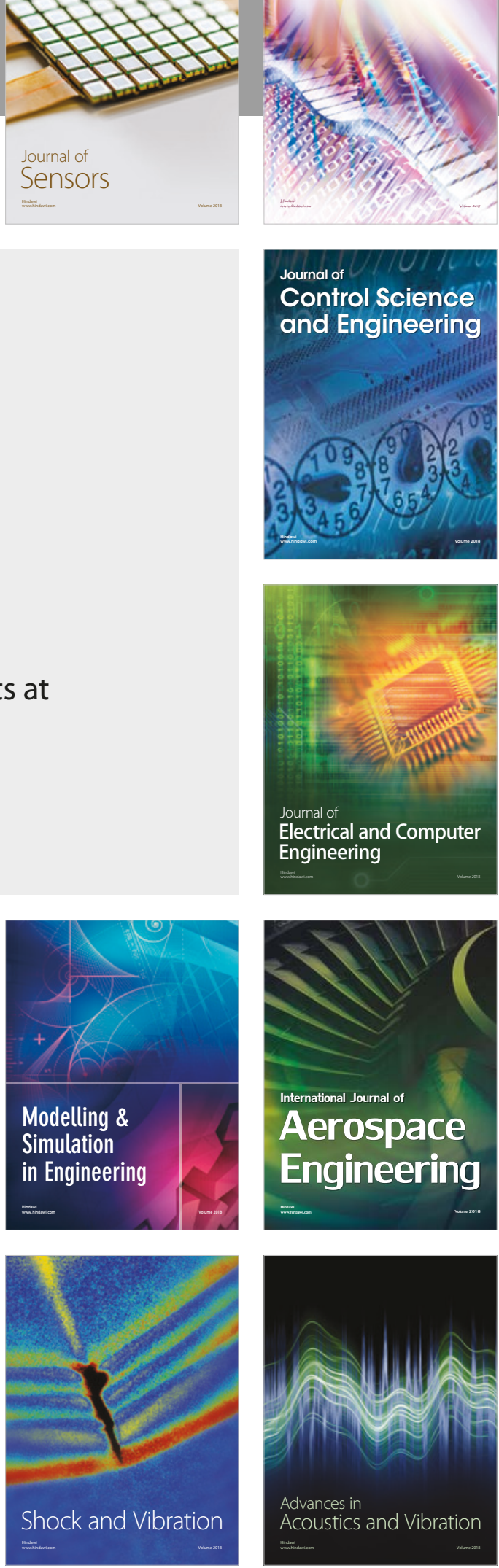